\title{
REAÇÕES DE OXIDAÇÃO DE DERIVADOS DE CIS-OCTALINAS PROMOVIDAS POR TRINITRATO DE TÁLIO (TTN)*
}

\author{
Helena M. C. Ferraz ${ }^{\dagger}$, Vânia M. T. Carneiro, Tiago O. Vieira e Luiz F. Silva Jr.*
}

Instituto de Química, Universidade de São Paulo, CP 26077, 05513-970 São Paulo - SP, Brasil

Recebido em 9/1/08; aceito em 4/3/08; publicado na web em 9/4/08

\begin{abstract}
OXIDATION REACTIONS OF DERIVATIVES OF CIS-OCTALINS PROMOTED BY THALLIUM TRINITRATE (TTN). The reaction of ten cis-octalins and cis-octalones with thallium trinitrate (TTN) leads to different products, depending mainly on the substitution pattern of the substrate. Functionalized cis-hydrindanes were obtained from the reaction of 1,2,3,4,4a,5,8,8a-octahydro4a-methylnaphthalene and of 1,2,3,4,4a,5,8,8a-octahydro-4a,7-dimethylnaphthalene with TTN in acetonitrile, whereas a cyclic ether was formed treating 1,2,3,4,4a,5,8,8a-octahydro-6,8a-dimethylnaphthalen-1-ol with TTN in trimethylorthoformate (TMOF).
\end{abstract}

Keywords: thallium trinitrate; cis-hydrindanes; octalin.

\section{INTRODUÇÃO}

Muitos produtos naturais contêm um sistema cis-hidrindânico como importante característica de sua estrutura molecular. Exemplos de substâncias naturais com atividade biológica que possuem este arranjo de anéis são os membros da classe de sesquiterpenóides bacanos $^{1-4}$ e tapsanos (Figura 1), ${ }^{5,6}$ bem como alguns produtos naturais relacionados com eremofilanos. ${ }^{7,8}$

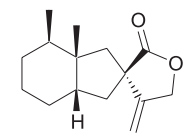

Baquenolida A

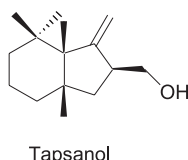

Tapsanol
Figura 1. Exemplos de produtos naturais contendo o sistema cis-hidrindânico

Diversas estratégias têm sido utilizadas para a construção do sistema cis-hidrindânico $^{9-23}$. Uma das clássicas abordagens é a condensação aldólica intramolecular de uma dicetona levando a uma hidrindanona que, após ser submetida a uma reação de hidrogenação catalítica, dá origem a um cis-hidrindano. ${ }^{9,10}$ Reações de Diels-Alder intermoleculares ${ }^{11-15}$ ou intramoleculares ${ }^{16}$ ocupam um lugar de destaque na construção de sistemas cishidrindânicos. Também possui um papel importante a reação de cicloadição de dicloroceteno com dimetilciclo-hexenos, que foi utilizada como etapa chave na síntese de diversos bacanos. ${ }^{17} \mathrm{Na}$ última década, nosso grupo de pesquisa investigou algumas abordagens para a formação de cis- e trans-hidrindanos através de reações de contração de anel mediadas por trinitrato de tálio (TTN). ${ }^{24-27}$ A reação de trans-2-decalonas com TTN em diclorometano levou a trans-hidrindanos funcionalizados com excelente rendimento e diastereosseletividade. ${ }^{24}$ Contudo, quando cis-2-decalonas foram utilizadas como materiais de partida, os produtos de contração foram obtidos em baixa regio- e diastereosseletividade, provavelmente devido à flexibilidade do sistema cis. ${ }^{25}$ Recentemente, uma nova estratégia foi investigada utilizando as cis-octalinas $\mathbf{1}$ e $\mathbf{2}$ como

\footnotetext{
$\dagger$ In memoriam, 1948-2007

*e-mail: luizfsjr@iq.usp.br

\#Este artigo é dedicado à Profa. Helena M. C. Ferraz
}

substratos para a reação de contração de anel mediada por TTN em metanol ou trimetilortoformiato (TMOF), visando a síntese de bacanos. Esta nova rota permitiu a preparação regiosseletiva dos cis-hidrindanos 3 e 4, os quais puderam ser transformados de maneira diastereosseletiva em compostos contendo um carbono quaternário em C2, como 5 (Esquema 1). ${ }^{26}$ Considerando estes resultados decidimos realizar o estudo, aqui apresentado, sobre a reatividade de uma série de cis-octalinas e de cis-octalonas com TTN em diferentes condições reacionais.<smiles>CC1CC=CCC1C</smiles>

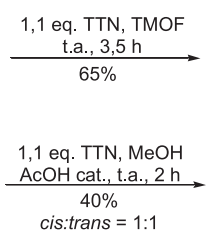<smiles>COC(C)C1CC(C)C2CCCCC2C1</smiles>
6 etapas<smiles>CC1=CCC2CCCCC2C1</smiles><smiles>CC(=O)C1C[C@H]2CCCC[C@]2(C)C1</smiles><smiles>CC(=O)C(CC1(C)CCC(C)CC1)(C(=O)O)C(=O)O</smiles>

Esquema 1

\section{RESULTADOS E DISCUSSÃO}

A construção do necessário sistema bicíclico foi realizada utilizando uma reação Diels-Alder entre a 2-metil-ciclo-hexenona $\mathbf{6}^{28}$ e o 1,3-butadieno ou 2-metil-1,3-butadieno, conforme descrito anteriormente. ${ }^{26} \mathrm{~A}$ partir das octalonas $\mathbf{7}$ e $\mathbf{8}$ foram preparados todos os outros substratos. Redução de Wolff-Kishner de $\mathbf{7}$ e $\mathbf{8}$ permitiu a obtenção de $\mathbf{1}$ e de $\mathbf{2}$, respectivamente (Esquema 2$).{ }^{26}$ A redução da cetona de $\mathbf{7}$ e de $\mathbf{8}$ foi realizada com $\mathrm{NaBH}_{4}$ de maneira distereosseletiva para os correspondentes álcoois secundários $9 \mathrm{e}$ 10 (Esquema 3). ${ }^{29}$

As funções oxigenadas foram protegidas com grupos muito utilizados em síntese orgânica. $\mathrm{O}$ álcool $\mathbf{1 0}$ foi transformado no correspondente acetato $11,{ }^{29}$ no éter de silício $12^{30}$ e no éter metílico 13 (Esquema 4). A preparação de 13 não foi possível utilizando uma condição clássica, com KOH/MeI em DMSO, ${ }^{31}$ na qual ocorreu a recuperação do material de partida. A carbonila da octalona 8 foi protegida na forma do correspondente acetal com etilenoglicol, fornecendo 14 (Esquema 5). Deste modo, um conjunto de dez octalinas e octalonas com diferentes grupos funcionais foi prepa- 


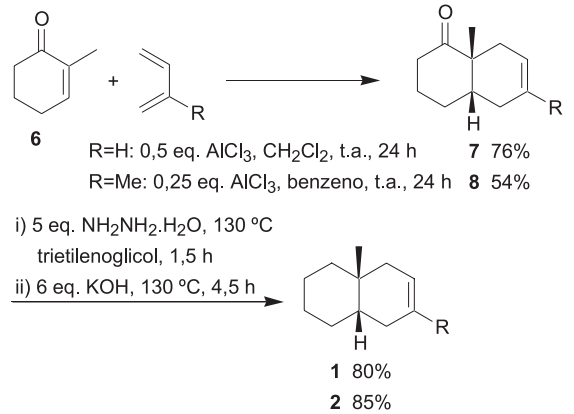

Esquema 2

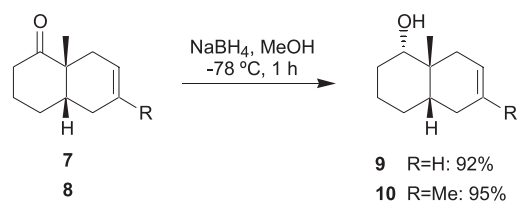

Esquema 3

rado e a reação de cada uma delas com tálio(III) foi investigada, conforme será discutido a seguir.

$$
\begin{aligned}
& 10 \stackrel{\substack{3 \text { eq. } \mathrm{Ac}_{2} \mathrm{O}, 20 \mathrm{~mol} \% \mathrm{DMAP}, \mathrm{E}_{3} \mathrm{~N}, \mathrm{t} . \mathrm{a}, 30 \mathrm{~min}}}{83 \%} \\
& 10 \frac{0,7 \text { eq. HMDS, } 9 \mathrm{~mol} \% \mathrm{KBr}}{\mathrm{MeCN} \text { t.a., } 30 \mathrm{~min}} \\
& 10 \underset{78 \%}{\substack{\text { i) } 2,5 \text { eq. BuLi, THF, } 0^{\circ} \mathrm{C}, 5 \mathrm{~min} \\
\text { ii) } 8 \mathrm{eq} \text {. Mel, t.a., } 16 \mathrm{~h}}}
\end{aligned}
$$

\section{Esquema 4}

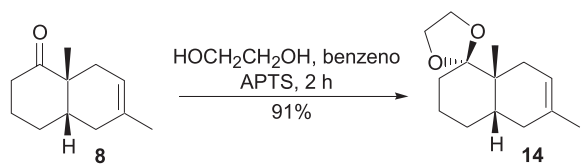

Esquema 5

Quando tratados com tálio(III), 3-alquenóis podem sofrer reações de ciclização, ${ }^{32-37}$ de rearranjo oxidativo (que podem levar a uma contração de anel), ${ }^{37-39}$ de fragmentação ${ }^{40,41}$ e de adição de solvente $^{37,42}$ (Esquema 6). No caso dos rearranjos oxidativos, a hidroxila desempenha um papel importante, alterando a reatividade da ligação dupla com TTN, já que o tálio(III) complexa com o oxigênio. ${ }^{43}$ Assim, decidimos investigar o comportamento do 4-alquenol 10 com TTN em trimetrilortoformiato (TMOF), que é a condição mais utilizada para promover uma reação de contração de anel. Ao realizar a reação neste meio, contudo, foi isolado exclusivamente o éter cíclico 15, em 63\% de rendimento (Esquema 7).

A formação do éter cíclico $\mathbf{1 5}$ ocorre por uma reação de ciclização eletrofílica mediada por tálio(III). A primeira etapa deve ser a formação do talônio 16, que sofreria um ataque da hidroxila, levando ao aduto oxitaliado 17 (caminho a). ${ }^{34}$ Considerando a provável conformação da molécula, a hidroxila estaria em uma posição bastante favorável para esta etapa intramolecular. Uma outra alternativa de mecanismo seria o talônio $\mathbf{1 6}$ fornecer um carbocátion

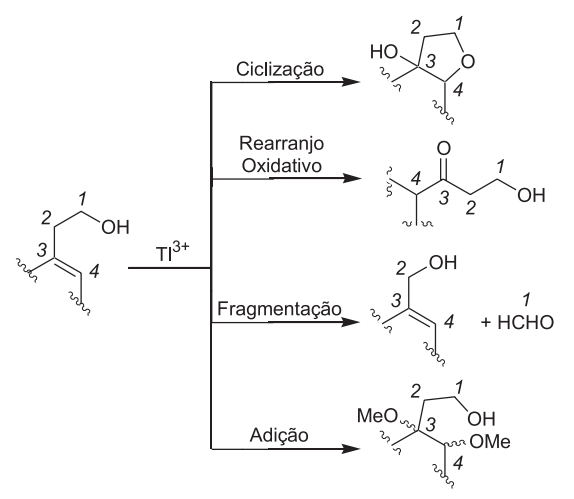

Esquema 6

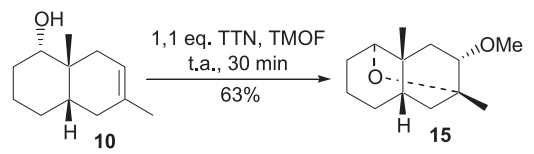

Esquema 7

terciário, no qual ocorreria o ataque intramolecular da hidroxila, fornecendo o aduto $\mathbf{1 7}$ (caminho b). Finalmente, a substituição do tálio(III) pelo grupo metoxila deve ocorrer por uma solvólise redutiva, levando a 15 (Esquema 8). Embora a reação de $\mathbf{1 0} \mathrm{com}$ tálio(III) tenha sido realizada com o objetivo do produto de contração de anel, o éter $\mathbf{1 5}$ obtido possui um arranjo de três anéis, que não seria facilmente obtido por outros métodos.

Com base no resultado acima, planejamos manter uma função oxigenada, na qual um ataque intramolecular pelo oxigênio não fosse favorável. Substratos nestas condições são o acetato $\mathbf{1 1}$ e o

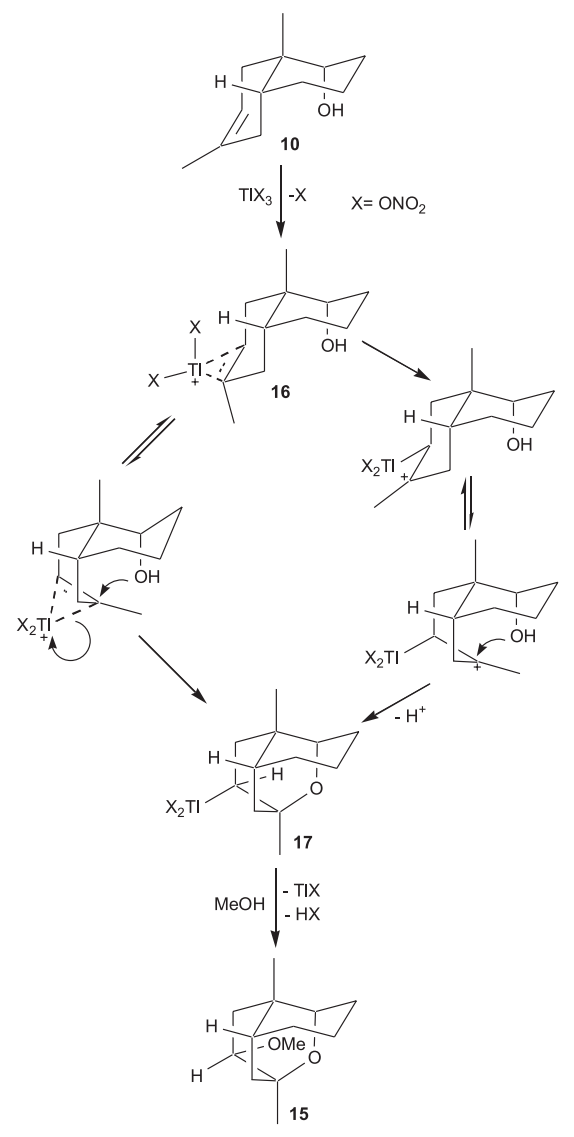

Esquema 8 
acetal 14. No caso do acetato insaturado 11, a reação com TTN em $\mathrm{MeOH}$ por $5 \mathrm{~min}$ a t.a. forneceu uma mistura complexa de diversos produtos de oxidação. A reação do acetal insaturado 14 forneceu dois produtos, sendo um deles de adição apenas do solvente (18), enquanto que o outro de adição de metanol e de nitrato (19) (Esquema 9). Embora estes compostos tenham sido isolados na forma de um único diastereoisômero, não foi possível determinar a configuração relativa.

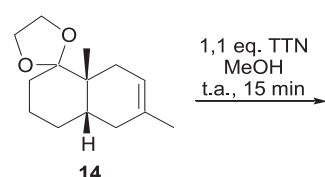

14
Esquema 9

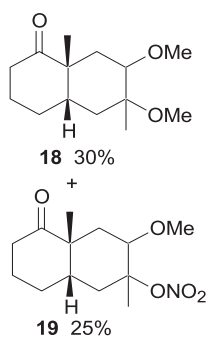

Recentemente, descobrimos que acetonitrila pode ser um excelente solvente para realizar reações de contração de anel de 1,2di-hidronaftalenos mediadas por iodo(III) ${ }^{44}$ Como iodo(III) possui uma reatividade similar à do tálio(III) e existem alguns relatos de rearranjos utilizando este mesmo solvente com sais de tálio(III), ${ }^{45,46}$ decidimos investigar o comportamento de cis-octalinas com tálio(III) em acetonitrila. Os primeiros substratos estudados foram as olefinas $\mathbf{1}$ e $\mathbf{2}$. A reação de $\mathbf{1}$ com TTN em acetonitrila forneceu o produto de contração de anel 20 em 50\% de rendimento, como uma mistura de diastereoisômeros na proporção de 9:2, cuja atribuição não foi realizada (Tabela 1, entrada 1). Embora o rendimento seja um pouco inferior ao observado em TMOF (65\%), utilizando acetonitrila é obtido o aldeído desprotegido (20), enquanto que em TMOF se isola o aldeído protegido na forma do acetal (3, Esquema 1). Dependendo da etapa subsequiente, a obtenção direta do aldeído pode ser vantajosa, por evitar a etapa de desproteção. Observou-se que o produto 20 sofreu oxidação ao ácido carboxílico correspondente após ser armazenado em geladeira. A reação da octalina 2 em acetonitrila forneceu o produto de contração de anel 4, com rendimento e diastereosseletividade comparáveis ao da reação em TMOF (entrada 2). Considerando que o comportamento das olefinas $\mathbf{1}$ e $\mathbf{2}$ foi semelhante tanto em acetonitrila quanto em $\mathrm{MeOH}$ ou TMOF, decidimos realizar a reação de $\mathbf{1 0}$ neste solvente, imaginando que o éter cíclico $\mathbf{1 5}$ fosse também formado. Contudo, quando $\mathbf{1 0}$ foi tratado com TTN em acetonitrila foi obtida uma mistura de diversos produtos, da qual foi possível isolar e caracterizar apenas o derivado glicólico 21, como uma mistura de diastereoisômeros na proporção de 5:1, em 20\% de rendimento (entrada 3). Em condições semelhantes, a reação da octalina 9 com tálio(III) também levou a um grande número de substâncias, embora neste caso não foi possível isolar na forma pura nenhum dos produtos de oxidação obtidos. A reação de 3-alquenóis protegidos na forma de éteres metílicos com tálio(III) forneceu o produto de contração de anel em bom rendimento. ${ }^{47}$ Deste modo, a reação do éter metílico insaturado $\mathbf{1 3}$ foi realizada com TTN em acetonitrila. Nesta condição, o produto de contração de anel desejado 22 foi isolado, porém em apenas $17 \%$ de rendimento (entrada 4). Embora 22 tenha sido isolado como um único diastereoisômero, não foi possível determinar a sua configuração relativa. Com o baixo rendimento desta reação, não é possível precisar se realmente ocorreu a formação de um único diastereoisômero ou se apenas um foi isolado. $\mathrm{O}$ grupo TMS não resiste às condições reacionais de oxidações com tálio(III). ${ }^{45,47}$ Porém, a proteção de alquenóis na forma de um éter de silício pode levar a melhores rendimentos no
Tabela 1. Oxidação de olefinas com 1,1 eq. de TTN. $3 \mathrm{H}_{2} \mathrm{O}$ em MeCN a $0{ }^{\circ} \mathrm{C}$ na presença de peneira molecular $3 \AA$

Entrada Substrato $\quad$ Produto (Rendimento)

1<smiles>CC12CC=CCC1CCCC2</smiles>

2

3

4

5

6<smiles>C[C@]12CC=CC[C@@H]1CCCC2=O</smiles>
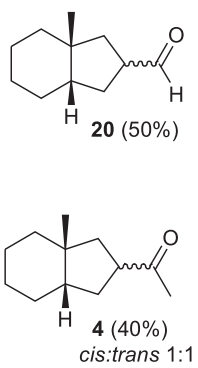<smiles>CC1=CCC2(O)C(C)CCCC2(C)C1</smiles>

$\mathrm{OH}$<smiles>CCC1CCCCC12CC(O)C(O)CC2(C)O</smiles>

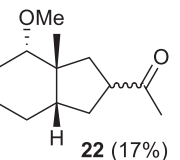

$\mathrm{OH}$<smiles>CC1=CCC2([N+](=O)[O-])CCCCC2(O)C1</smiles><smiles>O=C1CCC[C@@H]2C[C@H](O)C(O)CC12O</smiles><smiles>CC1=CCC2(C)C(=O)CCC[C@H]2C1</smiles>

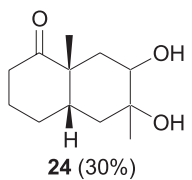

rearranjo oxidativo de olefinas. ${ }^{45}$ Realmente, a reação do alquenol protegido na forma de TMS (12) com tálio(III) foi mais limpa que a do substrato não protegido 10. Entretando, o produto formado não foi o de rearranjo, mas sim o de adição 21 (entrada 5), como uma mistura de diastereoisômeros, de maneira análoga ao observado com 10. Contudo, o rendimento da reação com 12 (54\%) foi superior ao da reação com 10 (20\%). Na reação das octalonas 7 e 8 com TTN em acetonitrila foi possível isolar os produtos de adição 23 e 24, respectivamente, ambos como um único diastereoisômero (entradas 6 e 7). Através da análise dos dados de RMN foi possível estabelecer a relação trans entre os grupos hidroxila e nitrato no produto 23. Uma explicação para o baixo rendimento destas duas últimas reações pode ser a presença de mais de um grupo funcional (cetona e olefina) oxidável pelo tálio(III).

Uma proposta de mecanismo para a formação dos produtos de contração de anel 1, 2 e 13 está exemplificada para 2 no Esquema 10. A primeira etapa deve ser a interação do tálio(III) com a ligação dupla, formando o íon talônio $\mathbf{2 5}$. A abertura trans-diaxial deste talônio ocorre pelo ataque de uma molécula de água, provenien- 


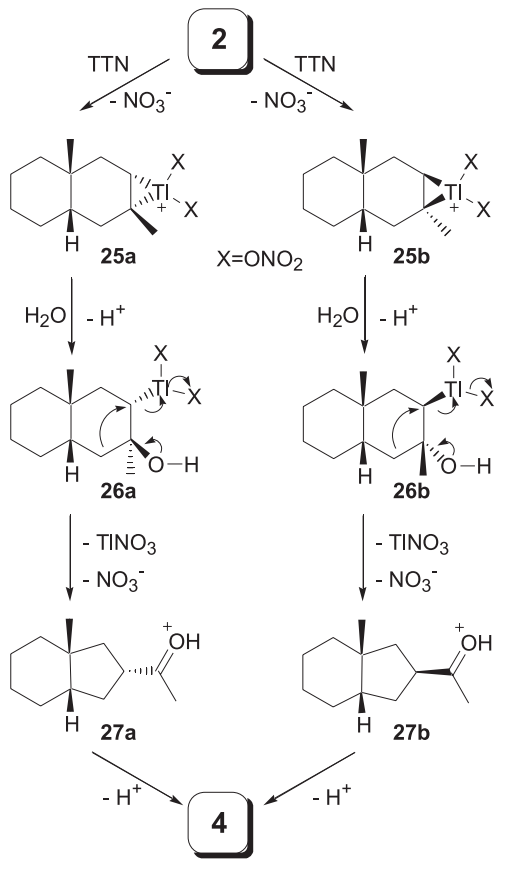

Esquema 10

te do sal de tálio(III) que é tri-hidrato, formando 26. Estas duas etapas ocorreriam por ambas as faces da ligação dupla, o que explica a baixa diasteresseletividade da reação. A etapa de rearranjo que leva a formação do anel de cinco membros ocorreria através da migração de um grupo alquila para o carbono ao qual está ligado o tálio(III), levando à cetona 4, após perda de próton de 27 (Esquema 10). Os produtos de adição a ligação dupla $(\mathbf{1 8}, \mathbf{1 9}, \mathbf{2 1}, 23$ e 24) devem ser originados por mecanismos análogos aos propostos para as reações de 1,2-di-hidronaftalenos com tálio(III). ${ }^{48}$

\section{PARTE EXPERIMENTAL}

\section{Informações gerais}

Todos os solventes e compostos químicos usados foram purificados de acordo com métodos usuais. As cromatografias em coluna foram feitas usando-se gel de sílica Acros 200-400 Mesh. As análises de CCD foram feitas com placas de gel de sílica Merck, e reveladas utilizando-se luz UV-254 nm e soluções de vanilina ou $p$-anisaldeído. Os espectros de $\mathrm{RMN}$ de ${ }^{1} \mathrm{H}$ e de ${ }^{13} \mathrm{C}$ foram feitos em $\mathrm{CDCl}_{3}$ com TMS como padrão interno e utilizando espectrômetros Bruker e Varian. Os espectros de IV foram medidos em espectrômetro Perkin-Elmer 1750FT. As análises de CG-EM foram feitas em um cromatógrafo a gás equipado com um analisador de massas Shimadzu 14B/QP5050A. Os espectros de massas de alta resolução foram feitos em espectrômetro Bruker Daltonics Microtof Eletrospray. Os pontos de fusão dos compostos sólidos foram determinados em aparelho de ponto de fusão Buchi B-545. Os substratos $\mathbf{1}^{26}, \mathbf{2}^{26}, \mathbf{7}^{26}, \mathbf{8}^{26}, \mathbf{9}^{29}, \mathbf{1 0}^{29}$, e $\mathbf{1 1}^{29}$ foram preparados de acordo com procedimentos descritos na literatura.

\section{(1,2,3,4,4a,5,8,8a-Octa-hidro-4a,7-dimetilnaftalen-4-ilóxi) trimetilsilano (12)}

A uma mistura do decalol 10 (0,190 g; 1,10 mmol) e $\mathrm{KBr}(0,05$ g; 0,1 mmol) em MeCN (3,0 mL), adicionou-se gota-a-gota HMDS $(0,30 \mathrm{~mL} ; 0,70 \mathrm{mmol})$. A mistura foi mantida sob agitação magnética a t.a. por $30 \mathrm{~min}$. O solvente foi evaporado sob pressão reduzida. Adicionou-se $\mathrm{H}_{2} \mathrm{O}(10 \mathrm{~mL})$ ao resíduo e fez-se extração com hexano $(2 \times 20 \mathrm{~mL})$. A fase orgânica foi seca com $\mathrm{MgSO}_{4}$ anidro e concentrada sob pressão reduzida obtendo-se $12(0,243 \mathrm{~g} ; 0,949$ mmol; 90\%), como um óleo incolor. IV (filme) $\left.v_{\max } / \mathrm{cm}^{-1}\right)$ : 2962 ; 2932; 2857; 1250; 1092; 838. RMN de ${ }^{1} \mathrm{H}\left(500 \mathrm{MHz}, \mathrm{CDCl}_{3}\right) \delta$ : $0,10(\mathrm{~s} ; 9 \mathrm{H}) ; 0,92(\mathrm{~s} ; 3 \mathrm{H}) ; 1,14-1,31(\mathrm{~m} ; 4 \mathrm{H}) ; 1,47-1,75(\mathrm{~m} ; 6 \mathrm{H})$; $1,62(\mathrm{~s} ; 3 \mathrm{H}) ; 2,14$ (d; $J=17,5 \mathrm{~Hz} ; 1 \mathrm{H}) ; 2,29$ (d; $J=17,5 \mathrm{~Hz} ; 1 \mathrm{H})$; $3,29$ (dd; $J=11,5$ e $5,0 \mathrm{~Hz} ; 1 \mathrm{H}) ; 5,27$ (s; $1 \mathrm{H})$. RMN de ${ }^{13} \mathrm{C}(126$ $\left.\mathrm{MHz}, \mathrm{CDCl}_{3}\right) \delta: 0,5 ; 23,7 ; 23,9 ; 24,2 ; 26,5 ; 28,9 ; 31,2 ; 34,4 ; 37,2$; 40,1; 78,8; 118,4; 130,2. EMBR (IE) $\mathrm{m} / \mathrm{z}$ (int. rel.) $252\left(\mathrm{M}^{+}, 3 \%\right.$ ); 162 (100); 147 (59); 119 (66); 106 (77); 105 (80); 91 (36); 74 (58); 73 (68). EMAR calc. para $\left[\mathrm{C}_{15} \mathrm{H}_{28} \mathrm{OSi}+\mathrm{Na}\right]^{+}: 275,1802$. Encontrado: 275,1803 .

\section{1,2,3,4,4a,5,8,8a-Octa-hidro-1-metóxi-6,8a-dimetilnaftaleno (13)}

A uma solução do decalol $\mathbf{1 0}(0,180 \mathrm{~g} ; 1,00 \mathrm{mmol})$ em THF anidro $(3,0 \mathrm{~mL})$, mantida em banho de gelo e sob atmosfera de $\mathrm{N}_{2}$, adicionou-se solução $2,5 \mathrm{M}$ de BuLi $(1,0 \mathrm{~mL} ; 2,5 \mathrm{mmol})$. A mistura foi mantida sob agitação por 5 min e adicionou-se MeI $(0,50$ $\mathrm{mL}$; $8,0 \mathrm{mmol}$ ). O banho de gelo foi removido e o sistema foi mantido sob agitação a t.a. por $16 \mathrm{~h}$. Adicionou-se $\mathrm{H}_{2} \mathrm{O}(10 \mathrm{~mL})$ à mistura reacional e fez-se extração da solução resultante com AcOEt (3 x $25 \mathrm{~mL})$. A fase orgânica foi lavada com solução saturada de $\mathrm{NaCl}(10 \mathrm{~mL})$, seca com $\mathrm{MgSO}_{4}$ anidro e concentrada sob pressão reduzida. O resíduo foi purificado por cromatografia flash (hexano/ AcOEt 6:1), obtendo-se o éter $\mathbf{1 3}$ (0,150 g; 0,773 mmol; 78\%), como um óleo incolor. IV (filme) $v_{\max } / \mathrm{cm}^{-1}$ ): 2964; 2930; 2857; 1466; 1102. RMN de ${ }^{1} \mathrm{H}\left(500 \mathrm{MHz}, \mathrm{CDCl}_{3}\right) \delta: 1,01$ (s; 3H); 1,18$1,33(\mathrm{~m} ; 5 \mathrm{H}) ; 1,49-1,54(\mathrm{~m} ; 1 \mathrm{H}) ; 1,58-1,62(\mathrm{~m} ; 1 \mathrm{H}) ; 1,62(\mathrm{~s} ; 3 \mathrm{H})$; $1,70-1,75(\mathrm{~m} ; 1 \mathrm{H}) ; 1,89-1,93(\mathrm{~m} ; 1 \mathrm{H}) ; 2,14-2,18(\mathrm{~m} ; 1 \mathrm{H}) ; 2,27-$ $2,31(\mathrm{~m} ; 1 \mathrm{H}) ; 2,77$ (dd, $J=11,0$ e 4,5 Hz; $1 \mathrm{H}) ; 3,33-3,37$ (m; 3H); $5,25-5,26(\mathrm{~m} ; 1 \mathrm{H})$. RMN de ${ }^{13} \mathrm{C}\left(75 \mathrm{MHz}, \mathrm{CDCl}_{3}\right) \delta: 23,7 ; 23,9$; 24,$0 ; 25,7 ; 26,7 ; 29,0 ; 34,0 ; 36,9 ; 40,4 ; 57,7 ; 87,8 ; 118,1 ; 130,2$. EMBR (IE) $m / z$ (int. rel.) $194\left(\mathbf{M}^{+}, 13 \%\right) ; 162$ (85); 147 (57); 119 (64); 106 (97); 105 (100); 91 (59). EMAR calc. para $\left[\mathrm{C}_{13} \mathrm{H}_{22} \mathrm{O}+\right.$ $\mathrm{Na}]^{+}:$217,1563. Encontrado: 217,1562.

\section{6,8a-Dimetil-3,4,4a,5,8,8a-hexa-hidro-2H-naftalen-1-ona- dixolano (14)}

Uma solução da cetona 8 (1,78 g; 10,0 mmol), etilenoglicol (1,86 g; 30,0 mmol) e ácido $p$-toluenossulfônico $(0,019$ g; $0,10 \mathrm{mmol})$ em benzeno anidro $(30 \mathrm{~mL})$ foi refluxada por $2 \mathrm{~h}$ em um aparelho de Dean-Stark. Após resfriamento, adicionou-se solução saturada de $\mathrm{NaHCO}_{3}(15 \mathrm{~mL})$ e fez-se extração da mistura resultante com AcOEt (3 x $50 \mathrm{~mL}$ ). A fase orgânica foi lavada com $\mathrm{H}_{2} \mathrm{O}(30 \mathrm{~mL})$, solução saturada de $\mathrm{NaCl}(30 \mathrm{~mL})$, seca com $\mathrm{MgSO}_{4}$ anidro e concentrada sob pressão reduzida. O resíduo foi purificado por cromatografia flash (hexano/AcOEt 75:25), obtendo-se 14 ${ }^{49}$ (2,02 g; 9,09 mmol; 91\%), como um óleo incolor. RMN de ${ }^{1} \mathrm{H}\left(300 \mathrm{MHz}, \mathrm{CDCl}_{3}\right) \delta: 0,87$ (s; $\left.3 \mathrm{H}\right)$; 1,20-1,80 (m; 9H); 1,63 (sl; 3H); 2,15-2,27 (m; 2H); 3,90-4,00 (m; $4 \mathrm{H}) ; 5,24-5,26$ (m; $1 \mathrm{H})$. RMN de ${ }^{13} \mathrm{C}\left(75 \mathrm{MHz}, \mathrm{CDCl}_{3}\right) \delta: 18,1 ; 22,9$; 23,$7 ; 28,6 ; 30,1 ; 30,4 ; 34,0 ; 38,2 ; 40,4 ; 64,9 ; 65,1 ; 113,0 ; 117,9 ; 130,5$.

\section{0-Metóxi-1,8-dimetil-2-oxa-triciclo[5.3.1.0] undecano (15)}

A uma solução do álcool $10(0,275 \mathrm{~g} ; 1,53 \mathrm{mmol})$ em TMOF (7,5 mL) adicionou-se TTN. $3 \mathrm{H}_{2} \mathrm{O}(0,75 \mathrm{~g} ; 1,68 \mathrm{mmol})$. Observou-se dissolução imediata do reagente seguida de precipitação abundante de um sólido branco a pós 1 min de reação. A mistura reacional foi mantida sob agitação a t.a. por 30 min e o término da reação foi verificado por CCD. A suspensão resultante foi filtrada, sob pressão, em uma coluna de gel de sílica $(15 \mathrm{~cm})$ usando $\mathrm{CH}_{2} \mathrm{Cl}_{2}(100 \mathrm{~mL})$ como eluente. A fase orgânica foi lavada com solução saturada de $\mathrm{NaCl}(15 \mathrm{~mL})$ e seca com $\mathrm{MgSO}_{4}$ anidro. O solvente foi removido sob pressão reduzida e o resíduo foi purificado por cromatografia flash (hexano/AcOEt 7:3), obtendo-se o 10-Metóxi-1,8-dimetil-2- 
oxa-triciclo[5.3.1.0] undecano 15 (0,202 g; 0,961 mmol; 63\%) como um óleo incolor. IV (filme) $\left.v_{\max } / \mathrm{cm}^{-1}\right): 1443 ; 1463$. RMN de ${ }^{1} \mathrm{H}(300$ $\left.\mathrm{MHz}, \mathrm{CDCl}_{3}\right) \delta: 0,86(\mathrm{~s} ; 3 \mathrm{H}) ; 1,15(\mathrm{~s} ; 3 \mathrm{H}) ; 1,24-1,33(\mathrm{~m} ; 4 \mathrm{H}) ; 1,45-$ $1,77(\mathrm{~m} ; 4 \mathrm{H}) ; 1,79-1,95(\mathrm{~m} ; 1 \mathrm{H}) ; 1,87(\mathrm{dd} ; J=13,6$ e $9,2 \mathrm{~Hz} ; 1 \mathrm{H})$; $2,07$ (dd; $J=14,0$ e $11,5 \mathrm{~Hz} ; 1 \mathrm{H}) ; 3,23$ (ddd; $J=9,3 ; 4,8$ e $1,9 \mathrm{~Hz}$; $1 \mathrm{H}) ; 3,33(\mathrm{~s} ; 3 \mathrm{H}) ; 3,55-3,57$ (m; $1 \mathrm{H})$. RMN de ${ }^{13} \mathrm{C}\left(75 \mathrm{MHz}, \mathrm{CDCl}_{3}\right)$ $\delta: 14,2 ; 22,0 ; 23,5 ; 26,7 ; 27,2 ; 32,5 ; 33,8 ; 33,8 ; 42,4 ; 70,2 ; 76,0$; 80,7. EMBR (IE) $\mathrm{m} / z$ (int. rel.) $210\left(\mathrm{M}^{+}, 45 \%\right) ; 94$ (100\%). Anal. calc. para $\mathrm{C}_{13} \mathrm{H}_{22} \mathrm{O}_{2}: \mathrm{C} 74,24 ; \mathrm{H} \mathrm{10,54}$. Encontrado: C 74,03; H 10,72.

\section{Oxidação de 14 com TTN em MeOH}

A uma solução do acetal $14(0,334 \mathrm{~g} ; 1,50 \mathrm{mmol}) \mathrm{em} \mathrm{MeOH}$ (7,5 mL) adicionou-se TTN. $3 \mathrm{H}_{2} \mathrm{O}(0,730 \mathrm{~g} ; 1,65 \mathrm{mmol})$. Observou-se a dissolução imediata do reagente seguida de precipitação abundante de um sólido branco após 1 min de reação. A mistura reacional foi mantida sob agitação a t.a. por 15 min e o término da reação foi verificado por CCD. A suspensão resultante foi filtrada, sob pressão, em uma coluna de gel de sílica $(15 \mathrm{~cm})$ usando $\mathrm{CH}_{2} \mathrm{Cl}_{2}$ $(100 \mathrm{~mL})$ como eluente. A fase orgânica foi lavada com solução saturada de $\mathrm{NaCl}(15 \mathrm{~mL})$ e seca com $\mathrm{MgSO}_{4}$ anidro. O solvente foi removido sob pressão reduzida e o resíduo foi purificado por cromatografia flash (eluição gradiente, 30-40\% de AcOEt em hexano), obtendo-se 18 (0,108 g; 0,450 mmol; $30 \%)$ e $\mathbf{1 9}(0,0833$ g; $0,307 \mathrm{mmol} ; 25 \%$ ) como sólidos brancos.

\section{6,7-dimetóxi-6,8a-dimetil-octa-hidronaftalen-1-ona (18)}

RMN de ${ }^{1} \mathrm{H}\left(200 \mathrm{MHz}, \mathrm{CDCl}_{3}\right) \delta: 0,87$ (dd; $J=13,2$ e $11,8 \mathrm{~Hz}$; $1 \mathrm{H}) ; 1,16(\mathrm{~s} ; 3 \mathrm{H}) ; 1,26(\mathrm{~s} ; 3 \mathrm{H}) ; 1,41-2,32(\mathrm{~m} ; 8 \mathrm{H}) ; 2,48-2,66(\mathrm{~m}$; $2 \mathrm{H}) ; 3,24$ (s; 3H); 3,33 (dd; $J=10,7$ e 4,8 Hz; $1 \mathrm{H}) ; 3,46$ (s; $3 \mathrm{H})$. $\mathrm{RMN}$ de ${ }^{13} \mathrm{C}\left(50 \mathrm{MHz}, \mathrm{CDCl}_{3}\right) \delta: 15,2 ; 22,0 ; 25,5 ; 26,6 ; 36,1 ; 37,4$; 37,$8 ; 41,8 ; 49,1 ; 49,4 ; 57,3 ; 77,8 ; 80,3 ; 214,0$.

\section{7-metóxi-6,8a-dimetil-6-nitróxi-octa-hidronaftalen-1-ona (19)}

IV (filme) $\left.\mathrm{v}_{\max } / \mathrm{cm}^{-1}\right): 1696 ; 1607 ; 1300 . \mathrm{RMN}$ de ${ }^{1} \mathrm{H}(200 \mathrm{MHz}$, $\left.\mathrm{CDCl}_{3}\right) \delta: 0,98(\mathrm{dd} ; J=13,6$ e $11,9 \mathrm{~Hz} ; 1 \mathrm{H}) ; 1,14-2,32(\mathrm{~m} ; 8 \mathrm{H})$; $1,28(\mathrm{~s} ; 3 \mathrm{H}) ; 1,59(\mathrm{~s} ; 3 \mathrm{H}) ; 2,52-2,62(\mathrm{~m} ; 2 \mathrm{H}) ; 3,42-3,54(\mathrm{~m} ; 1 \mathrm{H})$; $3,46(\mathrm{~s} ; 3 \mathrm{H})$. RMN de ${ }^{13} \mathrm{C}\left(50 \mathrm{MHz}, \mathrm{CDCl}_{3}\right) \delta: 15,4 ; 21,9 ; 25,3$; 26,$3 ; 36,3 ; 36,4 ; 37,3 ; 41,8 ; 49,0 ; 58,4 ; 78,3 ; 95,8 ; 213,4$.

Octa-hidro-3a-metil-1H-indano-2-carbaldeído (20). Procedimento geral para a oxidação de olefinas com tálio(III) em MeCN

A uma mistura da olefina $1(0,149 \mathrm{~g} ; 0,993 \mathrm{mmol})$ e peneira molecular $3 \AA$ ( $0,070 \mathrm{~g})$ em MeCN $(5,0 \mathrm{~mL})$, mantida em banho de gelo, adicionou-se TTN. $3 \mathrm{H}_{2} \mathrm{O}(0,460 \mathrm{~g} ; 1,09 \mathrm{mmol})$. A mistura reacional foi mantida sob agitação por 8 min e o término da reação foi verificado por CCD. A suspensão resultante foi filtrada, sob pressão, em uma coluna de gel de sílica $(15 \mathrm{~cm})$ usando $\mathrm{CH}_{2} \mathrm{Cl}_{2}(150 \mathrm{~mL})$ como eluente. A fase orgânica foi lavada com solução saturada de $\mathrm{NaCl}$ (15 mL) e seca com $\mathrm{MgSO}_{4}$ anidro. O solvente foi removido sob pressão reduzida e o resíduo foi purificado por cromatografia flash (hexano/AcOEt 5:1), obtendo-se o octa-hidro-3a-metil- $1 \mathrm{H}$-indano-2carbaldeído $2 \mathbf{2 0}^{26}$ (0,0830 g; 0,500 mmol; 50\%), como um óleo incolor e como uma mistura de diastereoisômeros, cuja proporção de 9:2 foi determinada por RMN. RMN de ${ }^{1} \mathrm{H}\left(300 \mathrm{MHz}, \mathrm{CDCl}_{3}\right) \delta$ : (diastereoisômero majoritário) 0,99 (s; 3H); 1,20-1,63 (m; 10H); 1,76$2,05(\mathrm{~m} ; 3 \mathrm{H}) ; 2,86-2,94(\mathrm{~m} ; 1 \mathrm{H}) ; 9,65(\mathrm{~d} ; J=2,1 \mathrm{~Hz} ; 1 \mathrm{H})$; (diastereoisômero minoritário) 1,01 (s; 3H); 1,20-1,63 (m; 10H); 1,762,05 (m; 3H); 2,86-2,94 (m; 1H); 9,70 (d; $J=2,1 \mathrm{~Hz} ; 1 \mathrm{H})$. RMN de ${ }^{13} \mathrm{C}\left(75 \mathrm{MHz}, \mathrm{CDCl}_{3}\right) \delta$ : (diastereoisômero majoritário) 21,8; 22,2; 25,6; 26,0; 29,4; 33,6; 39,5; 40,9; 44,4; 48,2; 204,0; (diastereoisômero minoritário) 22,2; 22,8; 26,8; 26,9; 30,2; 34,0; 38,0; 44,4; 44,8; 48,7; 204,5. O produto 20 sofreu oxidação ao ácido carboxílico correspondente após ser mantido em geladeira por 10 dias. IV (filme) $v_{\max } / \mathrm{cm}^{-1}$ ):
3300-2564; 1703; 1291; 1233. RMN de ${ }^{1} \mathrm{H}\left(300 \mathrm{MHz}, \mathrm{CDCl}_{3}\right) \delta$ : (diastereoisômero majoritário) 1,03 (s; 3H); 1,17-1,75 (m; 11H); 1,852,08 (m; 3H); 2,90-3,03 (m; 1H); (diastereoisômero minoritário) 0,98 (s; 3H); 11,17-1,75 (m; 11H); 1,85-2,08 (m; 3H); 2,90-3,03 (m; 1H). $\mathrm{RMN}$ de ${ }^{13} \mathrm{C}\left(75 \mathrm{MHz}, \mathrm{CDCl}_{3}\right) \delta$ : (diastereoisômero majoritário) 21,6; 22,$2 ; 25,3 ; 25,9 ; 32,6 ; 33,3 ; 39,5 ; 41,1 ; 43,4 ; 44,4 ; 183,5$; (diastereoisômero minoritário) 22,3; 23,0;26,8; 26,9; 33,5; 34,2; 39,9; 41,0; 44,4; 45,2; 183,3. EMBR (IE) $m / z$ (int. rel.) $182\left(\mathbf{M}^{+}, 4 \%\right.$ ); 110 (100); 95 (37); 81 (53); 67 (33). EMAR calc. para $\left[\mathrm{C}_{11} \mathrm{H}_{18} \mathrm{O}_{2}+\mathrm{Na}\right]^{+}$: 205,1199. Encontrado: 205,1197.

\section{1-(Octa-hidro-3a-metil-1H-inden-2-il)etanona (4)}

A reação foi feita seguindo o procedimento geral, mas usando a olefina $2(0,0911 \mathrm{~g} ; 0,555 \mathrm{mmol})$, peneira molecular $3 \AA(0,040$ g), $\mathrm{MeCN}(5,0 \mathrm{~mL})$ e TTN. $3 \mathrm{H}_{2} \mathrm{O}(0,255 \mathrm{~g} ; 0,605 \mathrm{mmol})$. O tempo de reação foi de 10 min e o resíduo foi purificado por cromatografia flash (hexano/AcOEt 9:1), obtendo-se a 1-(octa-hidro-3a-metil-1Hinden-2-il)etanona $4^{26}(0,0394 \mathrm{~g}$; 0,220 mmol; 40\%), como um óleo incolor e como uma mistura dos diastereoisômeros cis:trans 1:1, cuja proporção foi determinada por RMN.

\section{Deca-hidro-6,8a-dimetilnaftaleno-1,6,7-triol (21) a partir de 10}

A reação foi feita seguindo o procedimento geral, mas usando o álcool 10 (0,180 g; 1,00 mmol), peneira molecular $3 \AA$ (0,070 g), $\mathrm{MeCN}(5,0 \mathrm{~mL})$ e TTN. $3 \mathrm{H}_{2} \mathrm{O}(0,463 \mathrm{~g} ; 1,10 \mathrm{mmol})$. O tempo de reação foi de 5 min e o resíduo foi purificado por cromatografia flash (hexano/AcOEt 2:1), obtendo-se o deca-hidro-6,8a-dimetilnaftaleno1,6,7-triol 21 (0,0430 g; 0,201 mmol; 20\%), como um sólido branco. $\mathrm{O}$ produto $\mathbf{2 1}$ foi obtido como uma mistura de dois diastereoisômeros, cuja proporção de 5:1 foi estimada por CG. Pf: $93,4-94,1^{\circ} \mathrm{C}$. IV (KBr) $\left.v_{\max } / \mathrm{cm}^{-1}\right): 3444 ; 2937 ; 2859 ; 1444 ; 1067$. RMN de ${ }^{1} \mathrm{H}(300 \mathrm{MHz}$, $\left.\mathrm{CDCl}_{3}\right) \delta: 0,86(\mathrm{~s} ; 3 \mathrm{H}) ; 1,15(\mathrm{~s} ; 3 \mathrm{H}) ; 1,23-1,40(\mathrm{~m} ; 4 \mathrm{H}) ; 1,46-1,93(\mathrm{~m} ;$ $8 \mathrm{H}) ; 1,98$ (dd; $J=14,0$ e 9,8 Hz; $1 \mathrm{H}) ; 2,14$ (dd; $J=14,1$ e 11,4 Hz; $1 \mathrm{H}) ; 3,56-3,59$ (m; $1 \mathrm{H}) ; 3,74$ (ddd; $J=9,8 ; 5,0$ e 2,$0 ; 1 \mathrm{H})$. RMN de ${ }^{13} \mathrm{C}$ (75 MHz, $\mathrm{CDCl}_{3}$ ) $\delta$ : (diastereoisômero majoritário) 14,2; 21,8; 22,9; 26,7; 27,2; 31,4; 31,7; 34,0; 44,9; 70,8; 71,9; 76,0; (diastereoisômero minoritário) 14,$3 ; 20,7 ; 24,7 ; 26,5 ; 28,9 ; 34,3 ; 37,9 ; 38,0 ; 39,9 ; 73,7$; 81,3; 82,2. EMBR (IE) $m / z$ (int. rel.) (diastereoisômero majoritário) 196 (M+- 18, $71 \%$ ); 139 (47); 109 (38); 94 (100); 43 (98); (diastereoisômero minoritário) $196\left(\mathrm{M}^{+}-18,29 \%\right) ; 121$ (52); 108 (47); 95 (80); 43 (100). EMAR calc. para $\left[\mathrm{C}_{12} \mathrm{H}_{22} \mathrm{O}_{3}-\mathrm{H}_{2} \mathrm{O}+\mathrm{Na}\right]^{+}: 219,1356$. Encontrado: 219,1353.

1-(Octa-hidro-4-metóxi-3a-metil-1H-inden-2-il)etanona (22)

A reação foi feita seguindo o procedimento geral, mas usando a olefina $13(0,120 \mathrm{~g} ; 0,619 \mathrm{mmol})$, peneira molecular $3 \AA(0,070$ g), $\mathrm{MeCN}(5,0 \mathrm{~mL})$ e TTN.3H $\mathrm{O}(0,287 \mathrm{~g} ; 0,681 \mathrm{mmol})$. O tempo de reação foi de 30 min e o resíduo foi purificado por cromatografia flash (hexano/AcOEt 6:1), obtendo-se a 1-(octa-hidro-4-metóxi3a-metil-1H-inden-2-il)etanona 22 (0,0230 g; 0,105 mmol; 17\%), como um óleo levemente amarelado. IV (filme) $v_{\max } / \mathrm{cm}^{-1}$ ): 2932 ; 2874; 2820; 1710; 1450; 1359; 1099. RMN de ${ }^{1} \mathrm{H}\left(300 \mathrm{MHz}, \mathrm{CDCl}_{3}\right)$ $\delta$ : $0,98-1,01(\mathrm{~m} ; 2 \mathrm{H}) ; 1,08(\mathrm{~s} ; 3 \mathrm{H}) ; 1,08-2,08(\mathrm{~m} ; 9 \mathrm{H}) ; 2,15(\mathrm{~s}$; $3 \mathrm{H}) ; 2,98-3,05(\mathrm{~m} ; 2 \mathrm{H}) ; 3,34$ (s; $3 \mathrm{H})$. RMN de ${ }^{13} \mathrm{C}\left(75 \mathrm{MHz}, \mathrm{CDCl}_{3}\right)$ $\delta: 22,8 ; 25,2 ; 25,4 ; 29,0 ; 29,5 ; 31,2 ; 33,3 ; 46,1 ; 47,5 ; 48,9 ; 57,3$; 86,1; 210,8. EMBR (IE) $m / z$ (int. rel.) $210\left(\mathbf{M}^{+}, 8 \%\right.$ ); 195 (20); 135 (75); 111 (45); 71 (68); 43 (100). EMAR calc. para $\left[\mathrm{C}_{13} \mathrm{H}_{22} \mathrm{O}_{2}+\right.$ $\mathrm{Na}]^{+}:$233,1512. Encontrado: 233,1509.

Deca-hidro-6,8a-dimetilnaftaleno-1,6,7-triol (21) a partir de 12

A reação foi feita seguindo o procedimento geral, mas usando a olefina $12(0,252 \mathrm{~g} ; 1,00 \mathrm{mmol})$, peneira molecular $3 \AA(0,070$ g), $\mathrm{MeCN}(5,0 \mathrm{~mL})$ e TTN. $3 \mathrm{H}_{2} \mathrm{O}(0,463 \mathrm{~g} ; 1,10 \mathrm{mmol}) . \mathrm{O}$ tempo 
de reação foi de 5 min e o resíduo foi purificado por cromatografia flash (hexano/AcOEt 2:1), obtendo-se o deca-hidro-6,8adimetilnaftaleno-1,6,7-triol 21 (0,115 g; 0,540 mmol; 54\%), como um sólido branco.

Octa-hidro-6-nitrato-7-hidróxi-8a-metilnaftalen-1(2H)-ona (23)

A reação foi feita seguindo o procedimento geral, mas usando a olefina $7(0,164 \mathrm{~g} ; 1,00 \mathrm{mmol})$, peneira molecular $3 \AA(0,070 \mathrm{~g})$, $\mathrm{MeCN}(5,0 \mathrm{~mL})$ e TTN. $3 \mathrm{H}_{2} \mathrm{O}(0,463 \mathrm{~g} ; 1,10 \mathrm{mmol})$. O tempo de reação foi de 8 min e o resíduo foi purificado por cromatografia flash (hexano/AcOEt 1:1), obtendo-se o octa-hidro-6-nitrato-7hidróxi-8a-metilnaftalen-1(2H)-ona 23 (0,0396 g; 0,200 mmol; $16 \%$ ), como um sólido branco. Pf: $139,6-140,5{ }^{\circ} \mathrm{C}$. IV (KBr) $v_{\text {max }} /$ $\left.\mathrm{cm}^{-1}\right)$ : $3361 ; 2942 ; 2889 ; 1689 ; 1628 ; 1278 ; 865$. RMN de ${ }^{1} \mathrm{H}(500$ $\left.\mathrm{MHz}, \mathrm{CDCl}_{3}\right) \delta: 1,31(\mathrm{~s} ; 3 \mathrm{H}) ; 1,49(\mathrm{dd} ; J=15,0$ e 4,5 Hz; $1 \mathrm{H})$; $1,58-1,63(\mathrm{~m} ; 1 \mathrm{H}) ; 1,71(\mathrm{dt} ; J=14,5$ e $4,5 \mathrm{~Hz} ; 1 \mathrm{H}) ; 1,91-2,05(\mathrm{~m}$; $3 \mathrm{H}) ; 2,11-2,26(\mathrm{~m} ; 2 \mathrm{H}) ; 2,32(\mathrm{dd} ; J=15,0$ e $4,5 \mathrm{~Hz} ; 1 \mathrm{H}) ; 2,37$ (dtd; $J=14,0 ; 5,0$ e $1,0 \mathrm{~Hz} ; 1 \mathrm{H}) ; 2,63(\mathrm{ddd} ; J=14,5 ; 9,5$ e 7,5 Hz; $1 \mathrm{H})$; $3,77$ (sl; $1 \mathrm{H}) ; 3,94-3,96(\mathrm{~m} ; 1 \mathrm{H}) ; 5,10-5,13(\mathrm{~m} ; 1 \mathrm{H})$. RMN de ${ }^{13} \mathrm{C}$ $\left(75 \mathrm{MHz}, \mathrm{CDCl}_{3}\right) \delta: 23,4 ; 25,9 ; 26,0 ; 27,0 ; 35,5 ; 40,0 ; 40,2 ; 49,3$; 65,8; 81,5; 218,9. EMBR (IE) $\mathrm{m} / z$ (int. rel.) 198 (M+- 45, 2\%); 180 (13); 124 (44); 111 (47); 55 (75); 41 (100). EMAR calc. para $\left[\mathrm{C}_{11} \mathrm{H}_{17} \mathrm{NO}_{5}+\mathrm{Na}\right]^{+}:$266,0999. Encontrado: 266,0996.

\section{Octa-hidro-6,7-di-hidróxi-6,8a-dimetilnaftaleno-1(2H)-ona (24)}

A reação foi feita seguindo o procedimento geral, mas usando a olefina $8(0,178 \mathrm{~g} ; 1,00 \mathrm{mmol})$, peneira molecular $3 \AA$ (0,070 g), $\mathrm{MeCN}(5,0 \mathrm{~mL})$ e TTN. $3 \mathrm{H}_{2} \mathrm{O}(0,463 \mathrm{~g} ; 1,10 \mathrm{mmol})$. O tempo de reação foi de 10 min e o resíduo foi purificado por cromatografia flash (AcOEt), obtendo-se o octa-hidro-6,7-di-hidróxi-6,8adimetilnaftaleno-1(2H)-ona 24 (0,0634 g; 0,300 mmol; 30\%), como um sólido branco. Pf: $89,6-90,7^{\circ} \mathrm{C}$. IV (KBr) $\left.v_{\text {max }} / \mathrm{cm}^{-1}\right)$ : 3393; 2956; 2918; 1684; 1077. RMN de ${ }^{1} \mathrm{H}\left(300 \mathrm{MHz}, \mathrm{CDCl}_{3}\right) \delta: 1,17$ (ddd; $J=$ $14,0 ; 3,3$ e $1,6 \mathrm{~Hz} ; 1 \mathrm{H}) ; 1,26(\mathrm{~s} ; 3 \mathrm{H}) ; 1,29(\mathrm{~s} ; 3 \mathrm{H}) ; 1,40-1,47$ (m; $1 \mathrm{H}) ; 1,71$ (dd; $J=14,0$ e $13,6 \mathrm{~Hz} ; 1 \mathrm{H}) ; 1,71$ (dd; $J=15,1$ e $3,7 \mathrm{~Hz}$; $1 \mathrm{H})$; 1,89-1,99 (m; 2H); 2,21-2,44 (m;6H); 2,69 (ddd; $J=14,3$; $13,2$ e 7,6 Hz; $1 \mathrm{H}) ; 3,44-3,47(\mathrm{~m} ; 1 \mathrm{H})$. RMN de ${ }^{13} \mathrm{C}(75 \mathrm{MHz}$, $\left.\mathrm{CDCl}_{3}\right) \delta: 23,1 ; 26,0 ; 27,4 ; 27,6 ; 35,5 ; 35,8 ; 38,2 ; 40,2 ; 49,0 ; 72,3$; 73,4. EMBR (IE) $m / z$ (int. rel.) $212\left(\mathrm{M}^{++}, 10 \%\right.$ ); 194 (12); 168 (19); 111 (43); 97 (33); 55 (29); 43 (100). EMAR calc. para $\left[\mathrm{C}_{12} \mathrm{H}_{20} \mathrm{O}_{3}+\right.$ $\mathrm{Na}]^{+}:$235,1305. Encontrado: 235,1303.

\section{CONCLUSÃO}

Em resumo, a preparação de uma série de octalinas e octalonas foi descrita. Estes resultados poderão ser úteis na síntese de terpenóides contendo o esqueleto cis-decalínico, tais como, os clerodanos, ${ }^{50,51}$ kalihinenos, ${ }^{52-54}$ telepoganos ${ }^{55}$ e eremofilanos. ${ }^{3,7,8,56}$ A reação de diversas octalinas e octalonas com tálio(III) leva a diferentes produtos, dependendo principalmente do padrão de substituição do substrato. Os resultados mais promissores para futuras aplicações aparentemente são a obtenção de cis-hidrindanos funcionalizados a partir dos substratos $\mathbf{1}$ e $\mathbf{2}$ e a síntese de um interessante éter cíclico, no caso da oxidação do 4-alquenol 10.

\section{MATERIAL SUPLEMENTAR}

Está disponibilizado gratuitamente em http:// quimicanova.sbq.org.br, na forma de arquivo PDF.

\section{AGRADECIMENTOS}

À FAPESP, ao CNPq e à CAPES pelo contínuo apoio financeiro.

\section{REFERÊNCIAS E NOTAS}

1. Abe, N.; Onoda, R.; Shirahata, K.; Kato, T.; Woods, M. C.; Kitahara, Y.; Tetrahedron Lett. 1968, 9, 369.

2. Naya, K.; Takagi, I.; Kawaguchi, Y.; Asada, Y.; Hirose, Y.; Shinoda, N.; Tetrahedron 1968, 24, 5871.

3. Bodensieck, A.; Kunert, O.; Haslinger, E.; Bauer, R.; Helv. Chim. Acta 2007, 90, 183.

4. Para uma revisão sobre bacanos, veja: Silva, L. F., Jr.; Synthesis 2001, 671.

5. Rasmussen, U.; Christensen, S. B.; Sandberg, F.; Planta Med. 1981, 43, 336.

6. Smitt, U. W.; Cornet, C.; Norup, E.; Christensen, S. B.; Phytochemistry 1990, 29, 873 .

7. Fei, D. Q.; Li, S. G.; Liu, C. M.; Wu, G.; Gao, K.; J. Nat. Prod. 2007, 70, 241.

8. Zhang, Z. X.; Lin, C. J.; Li, P. L.; Jia, Z. J.; Planta Med. 2007, 73, 585.

9. Evans, D. A.; Sims, C. L.; Tetrahedron Lett. 1973, 14, 4691.

10. Srikrishna, A.; Reddy, T. J.; Nagaraju, S.; Sattigeri, J. A.; Tetrahedron Lett. 1994, 35, 7841 .

11. Reddy, D. S.; Org. Lett. 2004, 6, 3345.

12. Reddy, D. S.; Palani, K.; Balasubrahmanyam, D.; Kamath, V. B.; Iqbal, J.; Tetrahedron Lett. 2005, 46, 5211.

13. Mehta, G.; Reddy, D. S.; J. Chem. Soc., Perkin Trans. 1 2000, 1399.

14. Mehta, G.; Ramesh, S. S.; Can. J. Chem. 2005, 83, 581.

15. Constantino, M. G.; Oliveira, K. T.; Polo, E. C.; Silva, G. V. J.; Brocksom, T. J.; J. Org. Chem. 2006, 71, 9880.

16. Back, T. G.; Payne, J. E.; Org. Lett. 1999, 1, 663.

17. Brocksom, T. J.; Coelho, F.; Depres, J. P.; Greene, A. E.; Lima, M. E. F.; Hamelin, O.; Hartmann, B.; Kanazawa, A. M.; Wang, Y.; J. Am. Chem. Soc. 2002, 124, 15313.

18. Miyashita, M.; Suzuki, T.; Yoshikoshi, A.; J. Am. Chem. Soc. 1989, 111, 3728.

19. Patel, H. A.; Singh, A. N.; Dev, S.; Tetrahedron 1984, 40, 1873.

20. Fleming, F. F.; Zhang, Z.; Wang, Q.; Steward, W.; J. Org. Chem. 2003, $68,7646$.

21. Toyota, M.; Asoh, T.; Matsuura, M.; Fukumoto, K.; J. Org. Chem. 1996, $61,8687$.

22. Stork, G.; Taber, D. F.; Marx, M.; Tetrahedron Lett. 1978, 19, 2445.

23. Audenhove, M. V.; Keukeleire, D.; Vandewalle, M.; Tetrahedron Lett. 1982, $21,1979$.

24. Ferraz, H. M. C.; Silva, L. F., Jr.; J. Org. Chem. 1998, 63, 1716.

25. Ferraz, H. M. C.; Silva, L. F., Jr.; J. Braz. Chem. Soc. 2001, 12, 548.

26. Ferraz, H. M. C.; Vieira, T. O.; Silva, L. F., Jr.; Synthesis 2006, 2748.

27. Para uma revisão sobre reações de contração de anel mediadas por tálio(III), veja: Ferraz, H. M. C.; Silva, L. F., Jr.; Quim. Nova 2000, 23, 216.

28. O composto 6 foi preparado em duas etapas (bromação/desidroalogenação) a partir da 2-metil-ciclo-hexanona, segundo procedimento descrito na literatura: Breton, G. W.; J. Org. Chem. 1997, 62, 8952; Das, B.; Venkateswarlu, K.; Mahender, G.; Mahender, I.; Tetrahedron Lett. 2005, 46, 3041; Hua, D. H.; Chen, Y.; Sin, H. S.; Maroto, M. J.; Robinson, P. D.; Newell, S. W.; Perchellet, E. M.; Ladesich, J. B.; Freeman, J. A.; Perchellet, J. P.; Chiang, P. K. J.; J. Org. Chem. 1997, 62, 6888.

29. Ferraz, H. M. C.; Vieira, T. O.; Andrade, L. H.; Porto, A. L. M.; Tetrahedron: Asymmetry 2006, 17, 1990.

30. Shirini, F.; Mollarazi, E.; Synth. Commun. 2006, 36, 1109.

31. Johnstone, R. A. W.; Rose, M. E.; Tetrahedron 1979, 35, 2169.

32. Byrd, J. E.; Halpern, J.; J. Am. Chem. Soc. 1973, 95, 2586.

33. Ferraz, H. M. C.; Brocksom, T. J.; Pinto, A. C.; Abla, M. A.; Zocher, D. T. H.; Tetrahedron Lett. 1986, 27, 811.

34. Ferraz, H. M. C.; Ribeiro, C. M. R.; Grazini, M. V. A.; Brocksom, T. J.; Brocksom, U.; Tetrahedron Lett. 1994, 35, 1497; Brocksom, U.; Toloi, A. P.; Brocksom, T. J.; J. Braz. Chem. Soc. 1996, 7, 237.

35. Ferraz, H. M. C.; Grazini, M. V. A.; Ribeiro, C. M. R.; Brocksom, U.; Brocksom, T. J.; J. Org. Chem. 2000, 65, 2606.

36. Ferraz, H. M. C.; Longo, L. S., Jr.; Zukerman-Schpector, J.; J. Org. Chem. 2002, 67, 3518.

37. Silva, L. F.; Craveiro, M. V.; Molecules 2005, 10, 1419.

38. Ferraz, H. M. C.; Longo, L. S., Jr; Zucherman-Schpector, J.; J. Org. Chem. 2002, 67, 3518 .

39. Ferraz, H. M. C.; Silva, L. F., Jr.; Tetrahedron 2001, 57, 9939.

40. Kocovský, P.; Pour, M.; J. Org. Chem. 1990, 55, 5580.

41. Kocovský, P.; Langer, V.; Gogoll, A.; J. Chem. Soc., Chem. Commun. 1990, 1026.

42. Ferraz, H. M. C.; Silva, L. F., Jr.; Tetrahedron 2001, 57, 9939.

43. Silva, L. F., Jr.; Quintiliano, S. A. P.; Craveiro, M. V.; Vieira, F. Y. M.; Ferraz, H. M. C.; Synthesis 2007, 355.

44. Silva, L. F., Jr.; Siqueira, F. A.; Pedrozo, E. C.; Vieira, F. Y. M.; Doriguetto, A. C.; Org. Lett 2007, 9, 1433. 
45. Kim, S.; Uh, K. H.; Tetrahedron Lett. 1996, 37, 3865.

46. Kaye, A.; Neidle, S.; Reese, C. B.; Tetrahedron Lett. 1988, 29, 1841

47. Ferraz, H. M. C.; Silva, L. F., Jr.; Synthesis 2002, 1033.

48. Silva, L. F., Jr.; Sousa, R. M. F.; Ferraz, H. M. C.; Aguilar, A. M.; J. Braz Chem. Soc. 2005, 16, 1160 .

49. Das, J.; Valenta, Z.; Liu, H.-J.; Ngooi, T.; Can. J. Chem. 1984, 62, 481

50. Anderson, A. B.; McCrindle, R.; Nakamura, E.; Chem. Commun. 1974 1974, 453.

51. Geis, W.; Buschauer, B.; Becker, H.; Phytochemistry 1999, 51, 643.
52. Okino, T.; Yoshimura, E.; Hirota, H.; Fusetani, N.; Tetrahedron Lett. 1995, $36,8637$.

53. Fusetani, N.; Yasumuro, K.; Kawai, H.; Natori, T.; Brinen, L.; Clardy, J.; Tetrahedron Lett. 1990, 31, 3599.

54. Rodriguez, J.; Nieto, R. M.; Hunter, L. M.; Diaz, M. C.; Crews, P.; Tetrahedron 1994, 50, 11079.

55. Shoji, N.; Umeyama, A.; Teranaka, M.; Arihara, S.; J. Nat. Prod. 1996, $59,448$.

56. Ishii, H.; Tozyo, T.; Minato, H.; Tetrahedron 1965, 21, 2605. 
REAÇÕES DE OXIDAÇÃO DE DERIVADOS DE CIS-OCTALINAS PROMOVIDAS POR TRINITRATO DE TÁLIO (TTN)\#

Helena M. C. Ferraz ${ }^{\dagger}$, Vânia M. T. Carneiro, Tiago O. Vieira e Luiz F. Silva Jr.*

Instituto de Química, Universidade de São Paulo, CP 26077, 05513-970 São Paulo - SP, Brasil

Foram selecionados espectros de RMN de ${ }^{1} \mathrm{H}$ e RMN de ${ }^{13} \mathrm{C}$ de alguns dos produtos obtidos após reação de oxidação dos derivados de cis-octalinas.

Para o produto $\mathbf{2 0}$ foram mostradas as atribuições do isômero majoritário.

As atribuições dos sinais em 15, 21, 23 e $\mathbf{2 4}$ foram feitas com o auxílio de RMN de COSY e HETCOR. Para o produto $\mathbf{2 1}$ foram mostradas as atribuições do isômero majoritário.

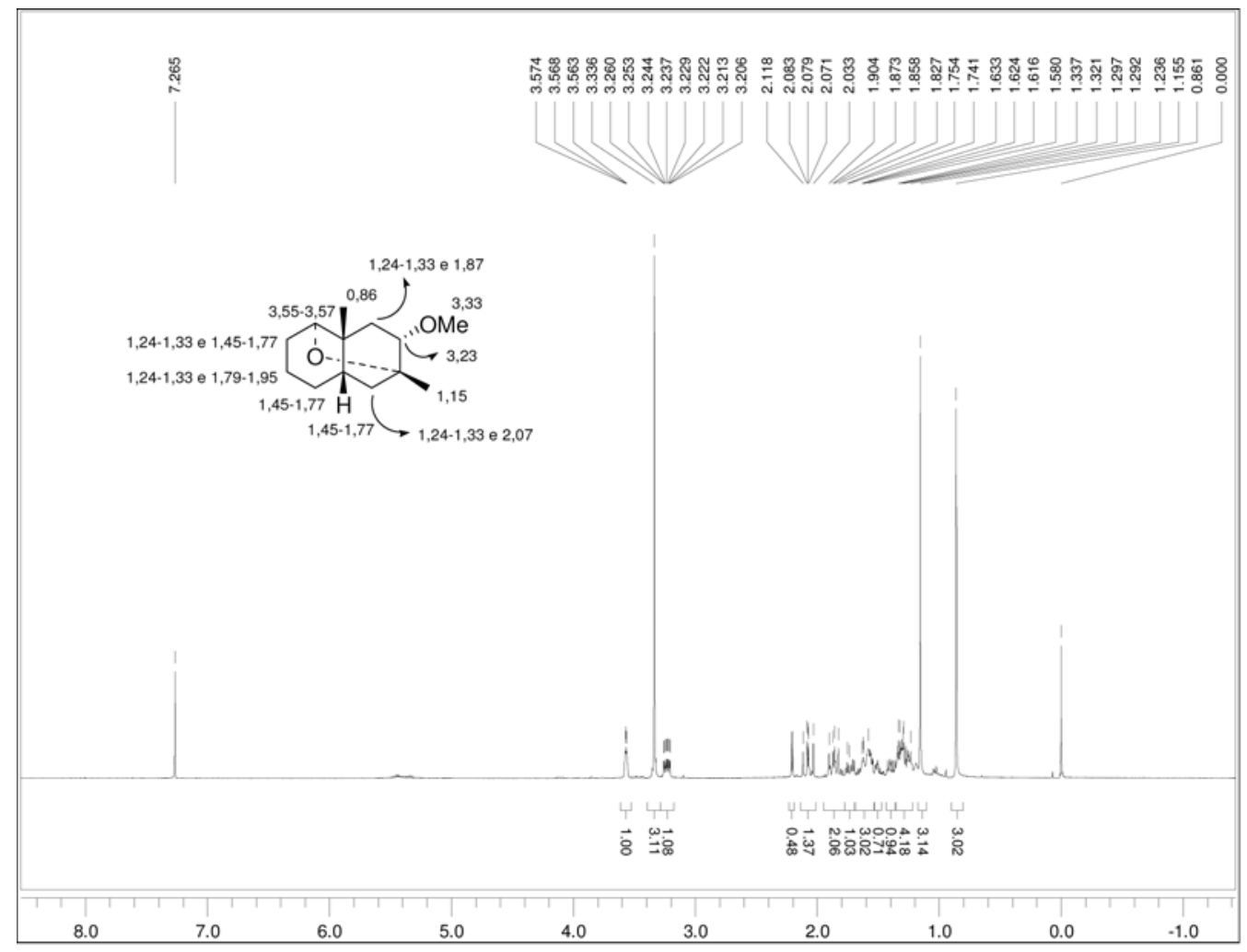

Figura 1S. Espectro de $\mathrm{RMN} \mathrm{de}{ }^{1} \mathrm{H}(300 \mathrm{MHz}), \mathrm{TMS}, \mathrm{CDCl}_{3}$ - Produto 15 

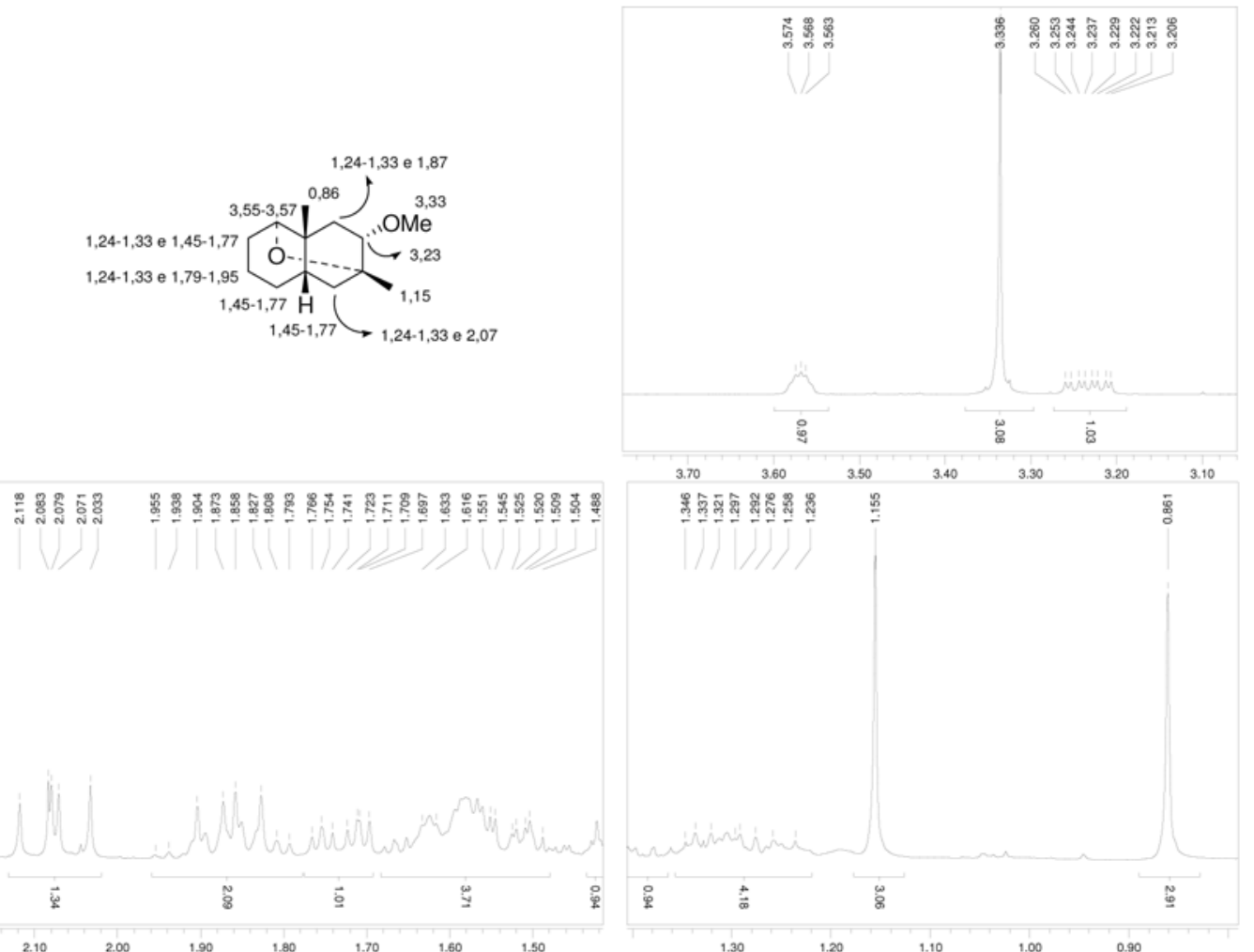

Figura 2S. Expansões do espectro de $\mathrm{RMN}$ de ${ }^{l} \mathrm{H}(300 \mathrm{MHz}), \mathrm{TMS}, \mathrm{CDCl}_{3}-$ Produto 15

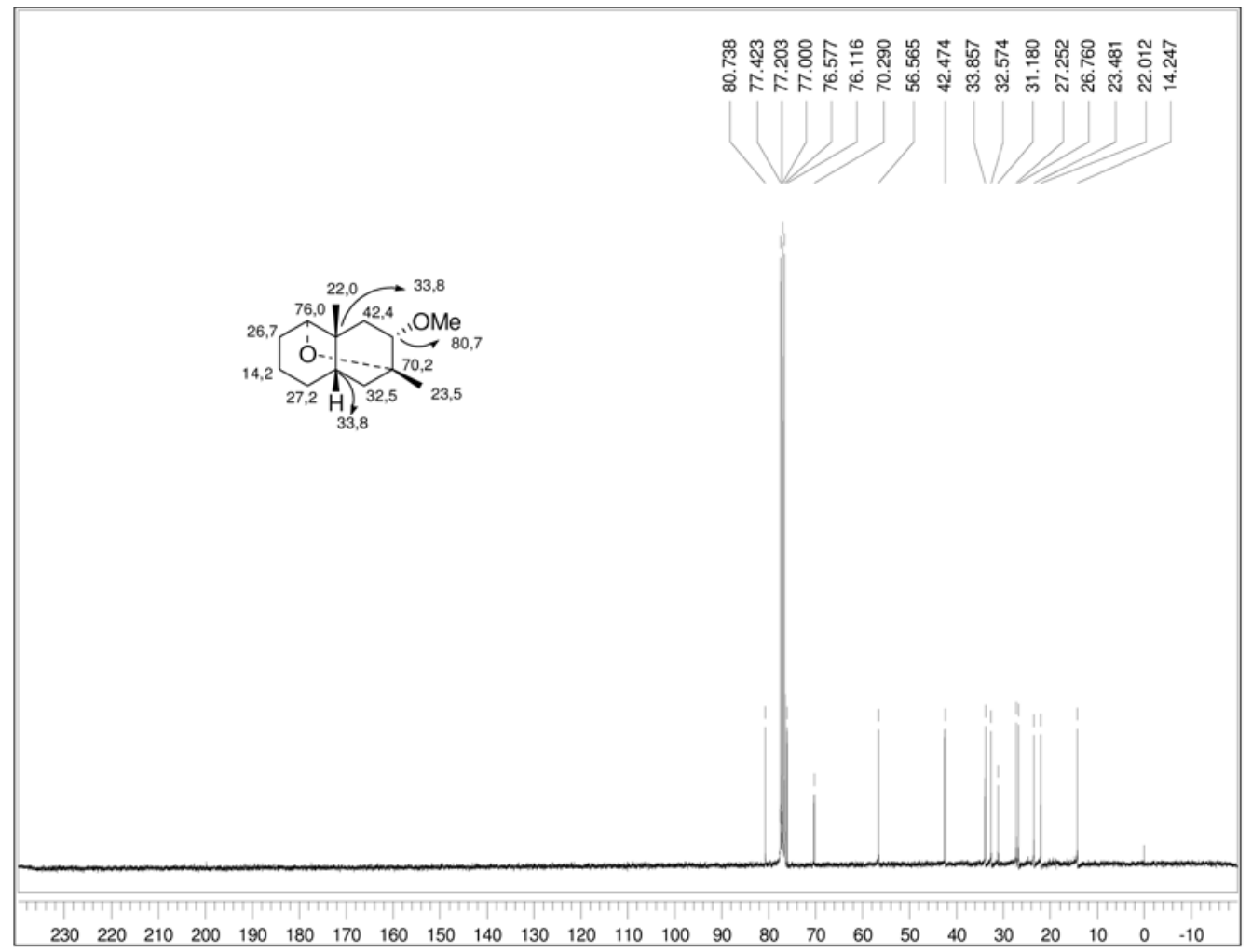

Figura 3S. Espectro de $\mathrm{RMN}$ de ${ }^{13} \mathrm{C}(75 \mathrm{MHz})$, TMS, $\mathrm{CDCl}_{3}$ - Produto 15 


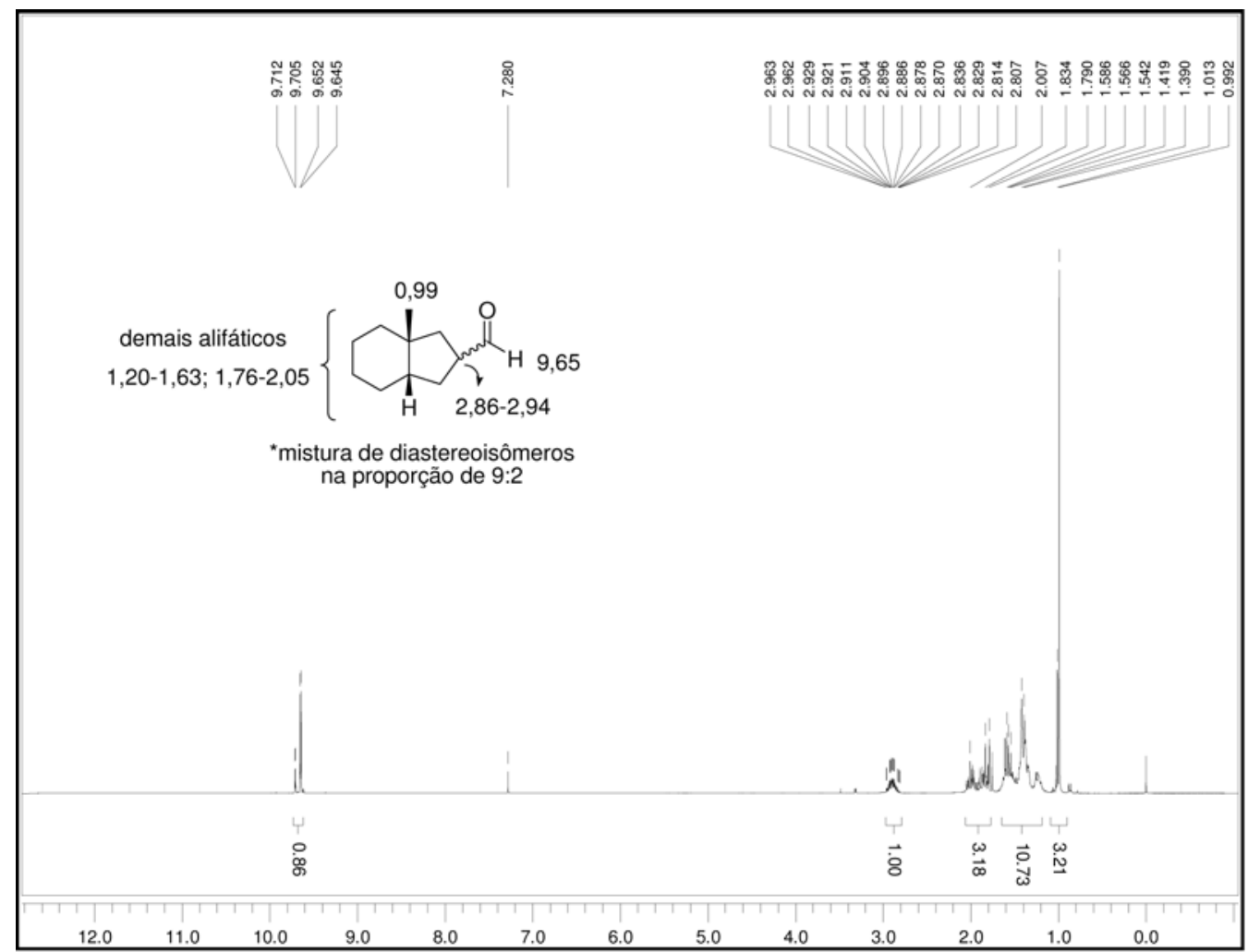

Figura 4S. Espectro de RMN de ${ }^{l} \mathrm{H}(300 \mathrm{MHz}), \mathrm{TMS}, \mathrm{CDCl} \mathrm{Cl}_{3}-$ Produto 20

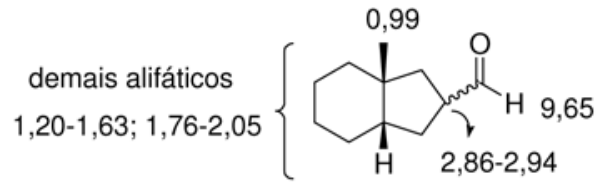

*mistura de diastereoisômeros

na proporção de 9:2

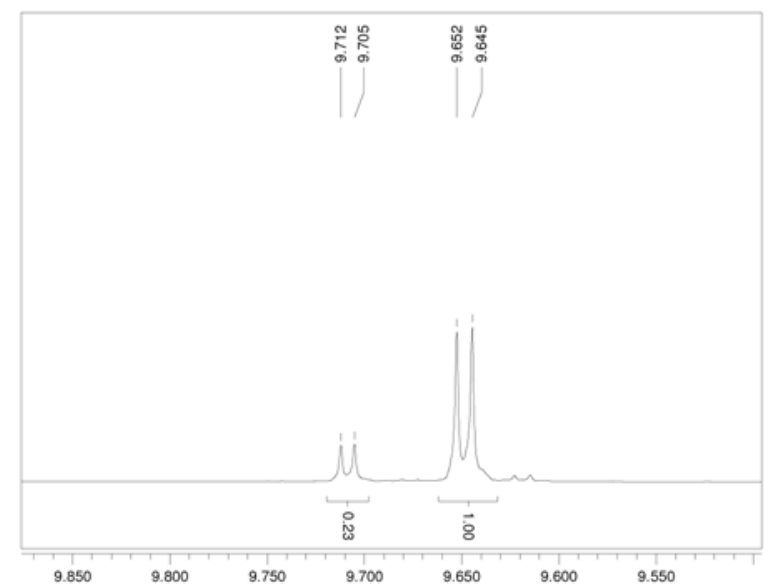

Figura 5S. Expansão do espectro de $\mathrm{RMN}$ de ${ }^{1} \mathrm{H}$ (300 MHz), TMS, CDCl 3 - Produto 20 


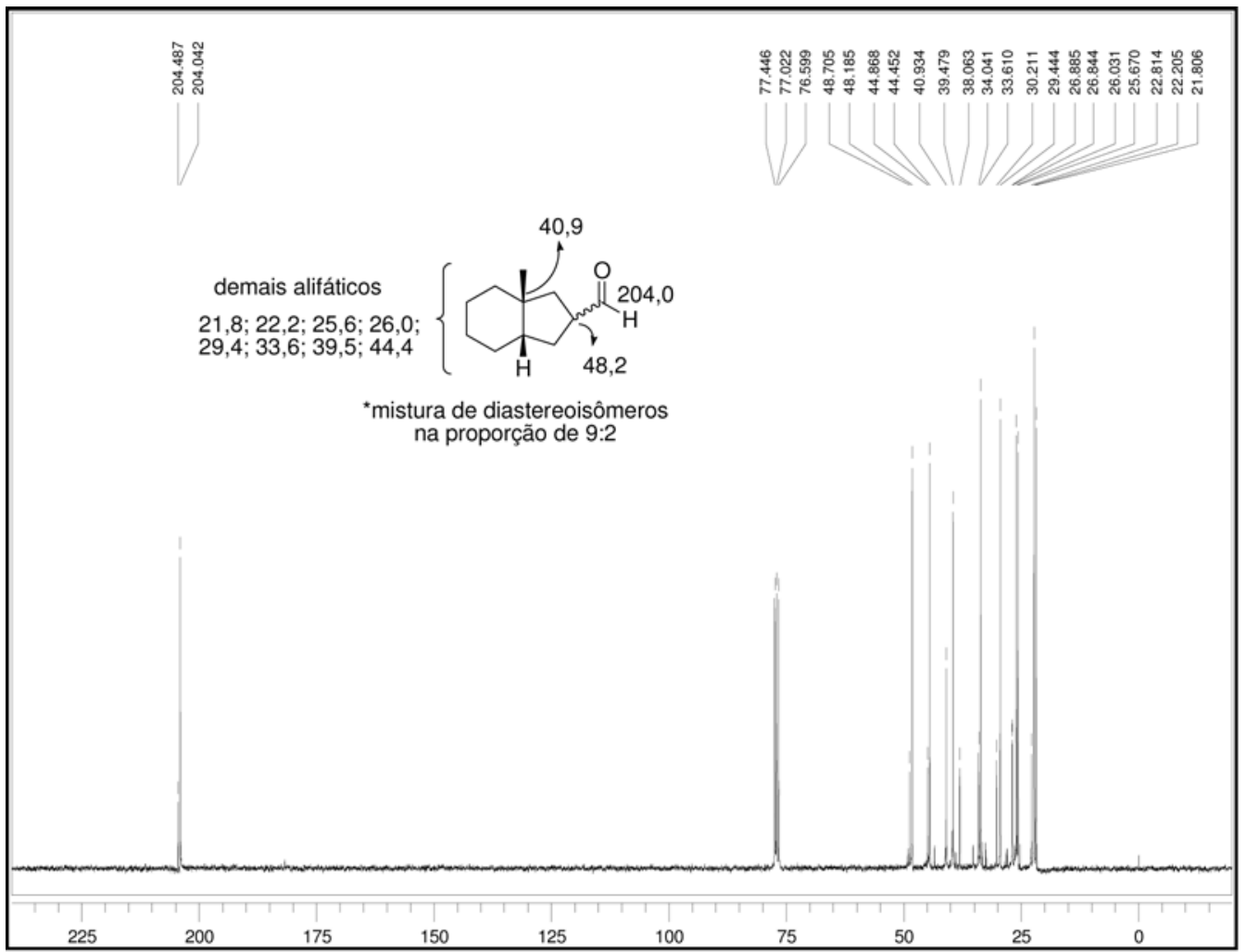

Figura 6S. Espectro de RMN de ${ }^{13} \mathrm{C}(75 \mathrm{MHz}), \mathrm{TMS}, \mathrm{CDCl}_{3}-$ Produto 20

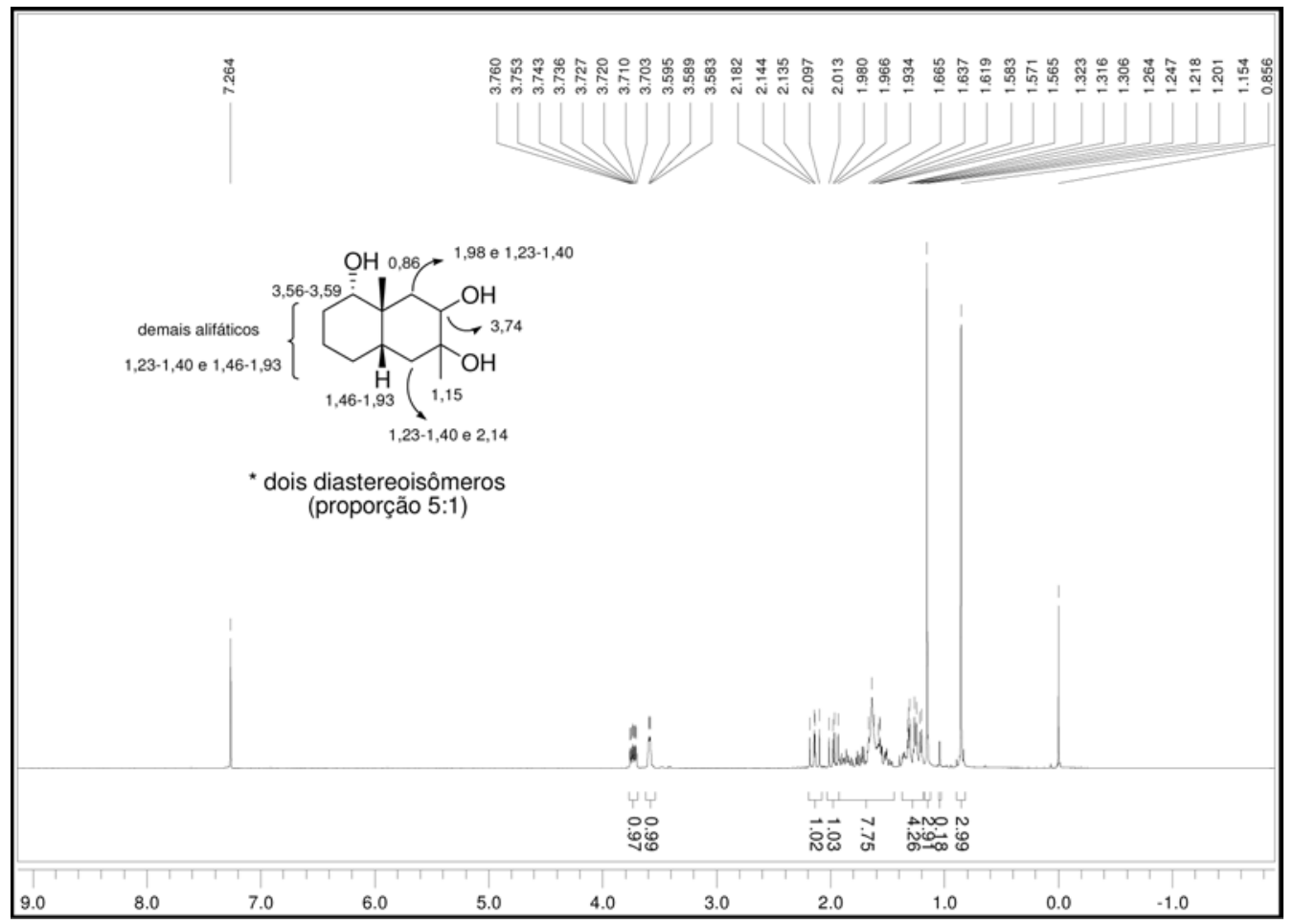

Figura 7S. Espectro de RMN de ${ }^{1} \mathrm{H}(300 \mathrm{MHz}), \mathrm{TMS}, \mathrm{CDCl}_{3}-$ Produto 21 

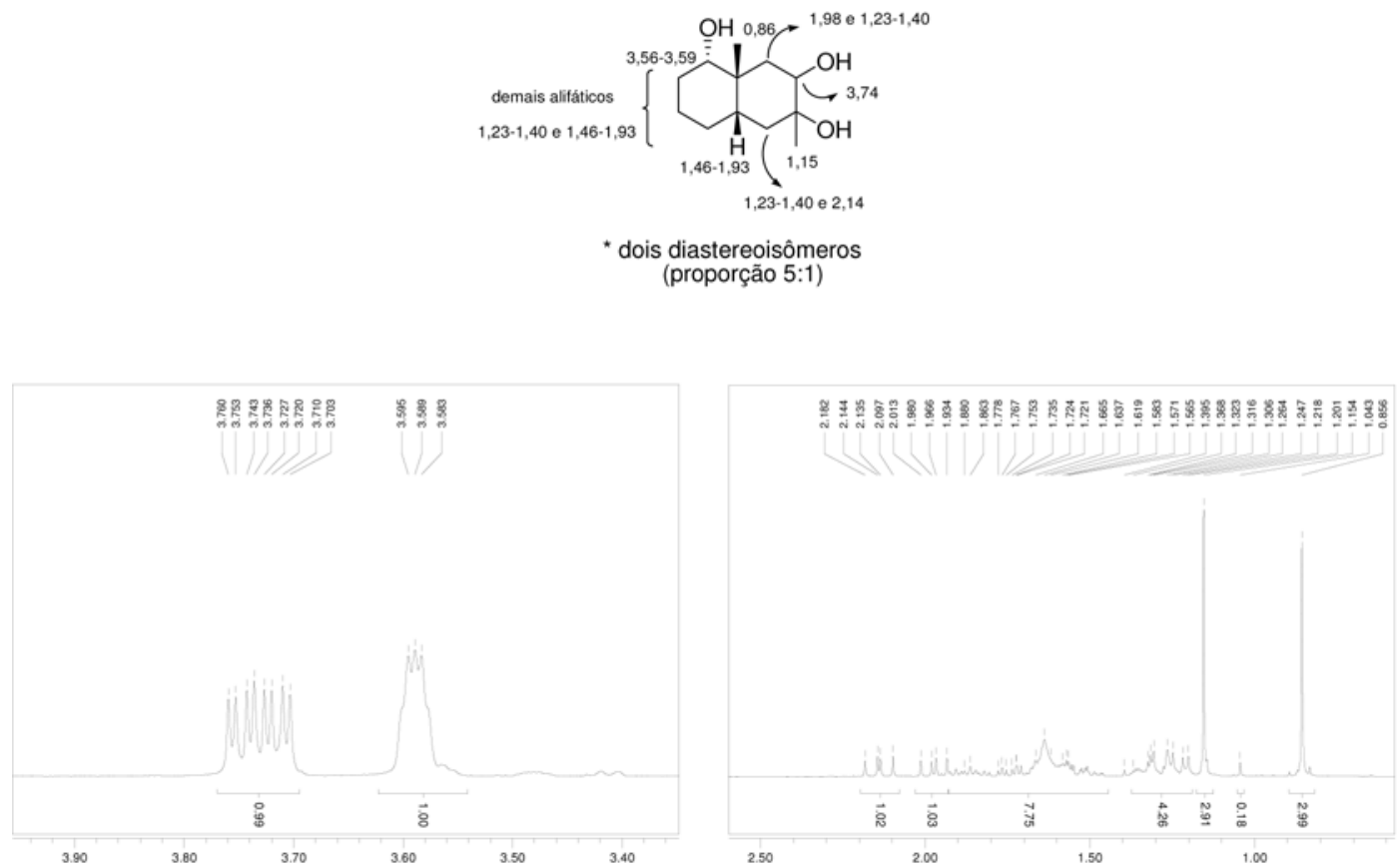

Figura 8S. Expansões do espectro de $\mathrm{RMN}$ de ${ }^{1} \mathrm{H}(300 \mathrm{MHz}), \mathrm{TMS}, \mathrm{CDCl}_{3}-$ Produto $^{21}$

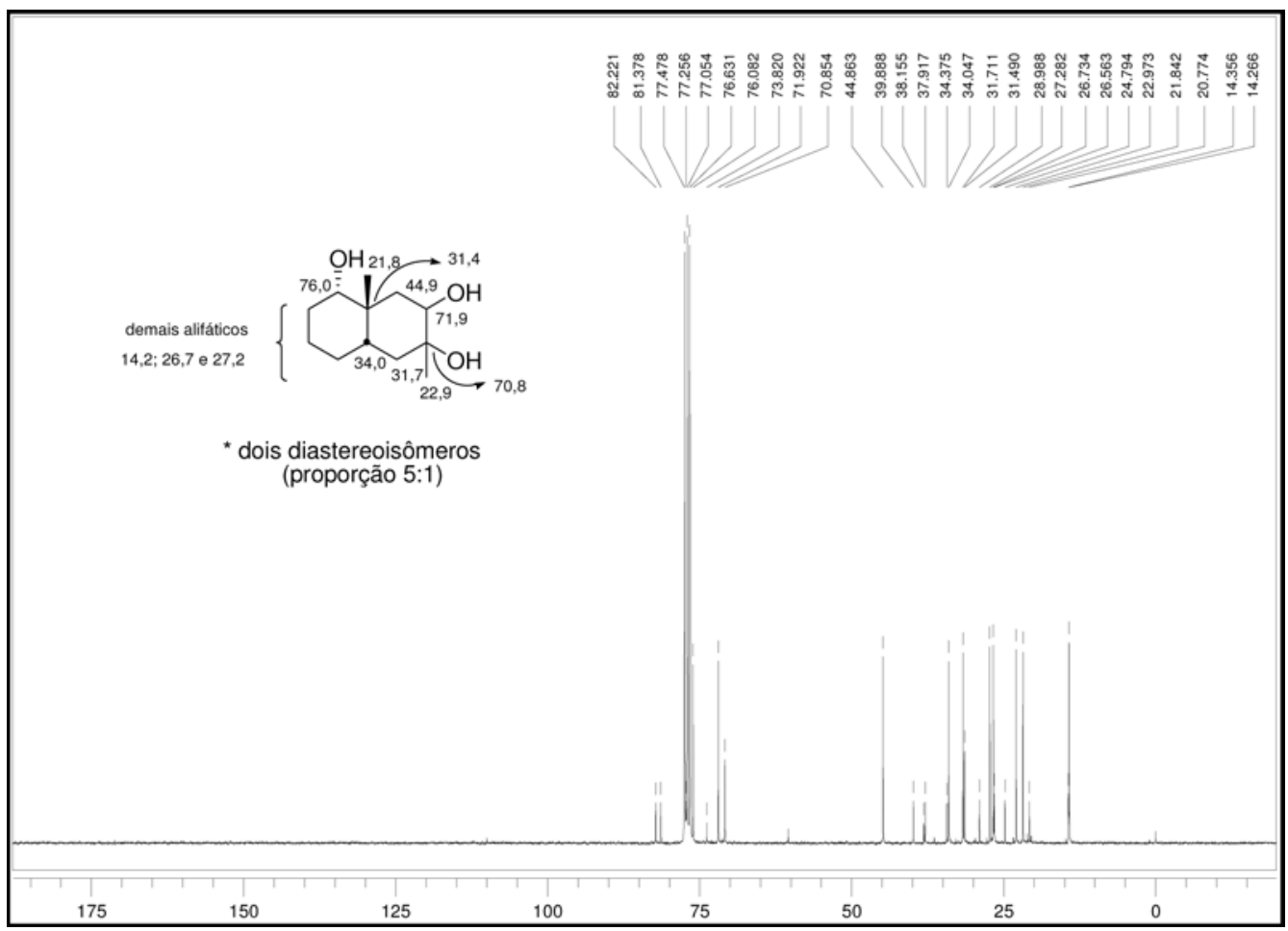

Figura 9S. Espectro de RMN de ${ }^{13} \mathrm{C}$ (75 $\left.\mathrm{MHz}\right), \mathrm{TMS}, \mathrm{CDCl}_{3}-$ Produto 21 


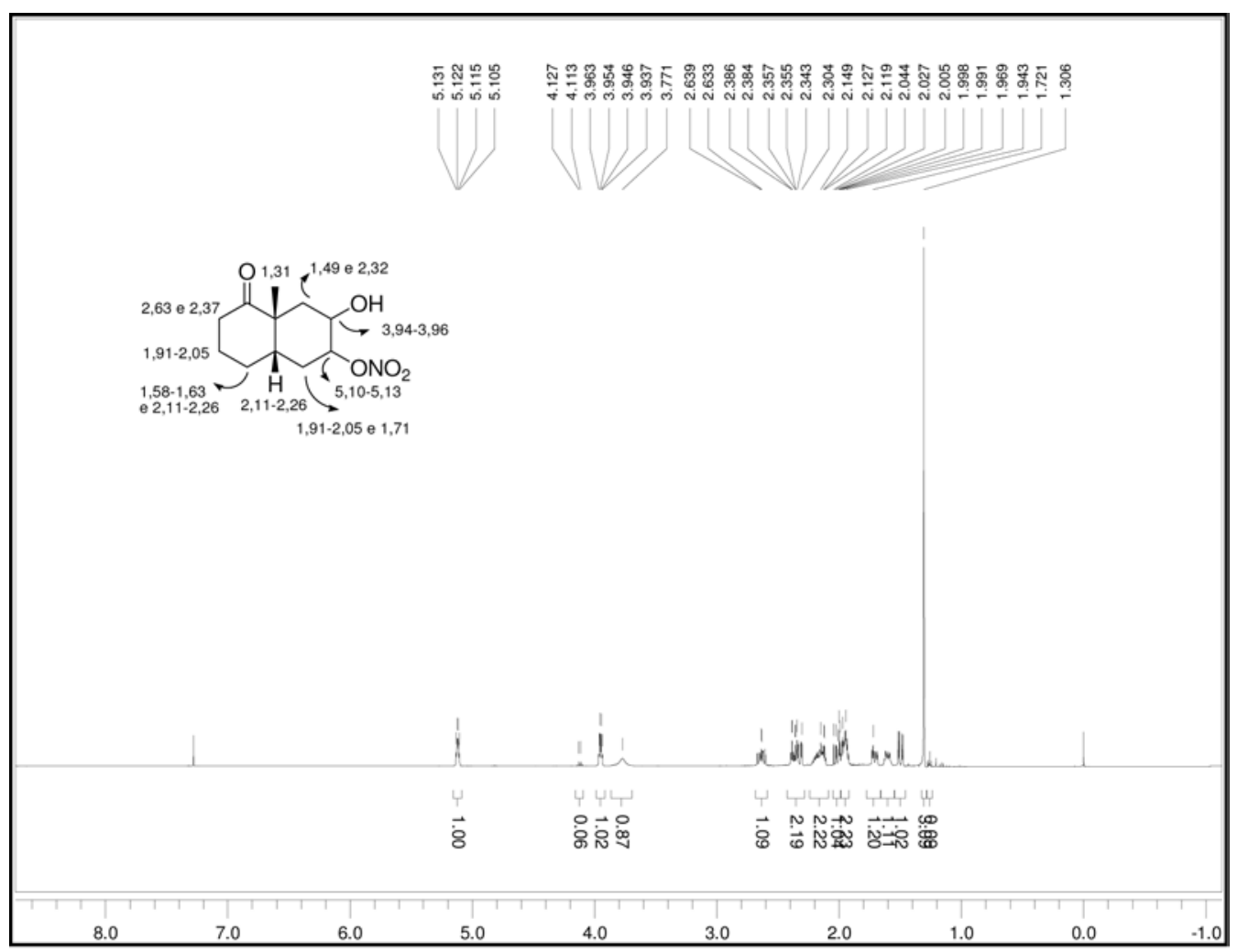

Figura 10S. Espectro de $\mathrm{RMN}$ de ${ }^{l} \mathrm{H}(500 \mathrm{MHz}), \mathrm{TMS}, \mathrm{CDCl}_{3}-$ Produto 23
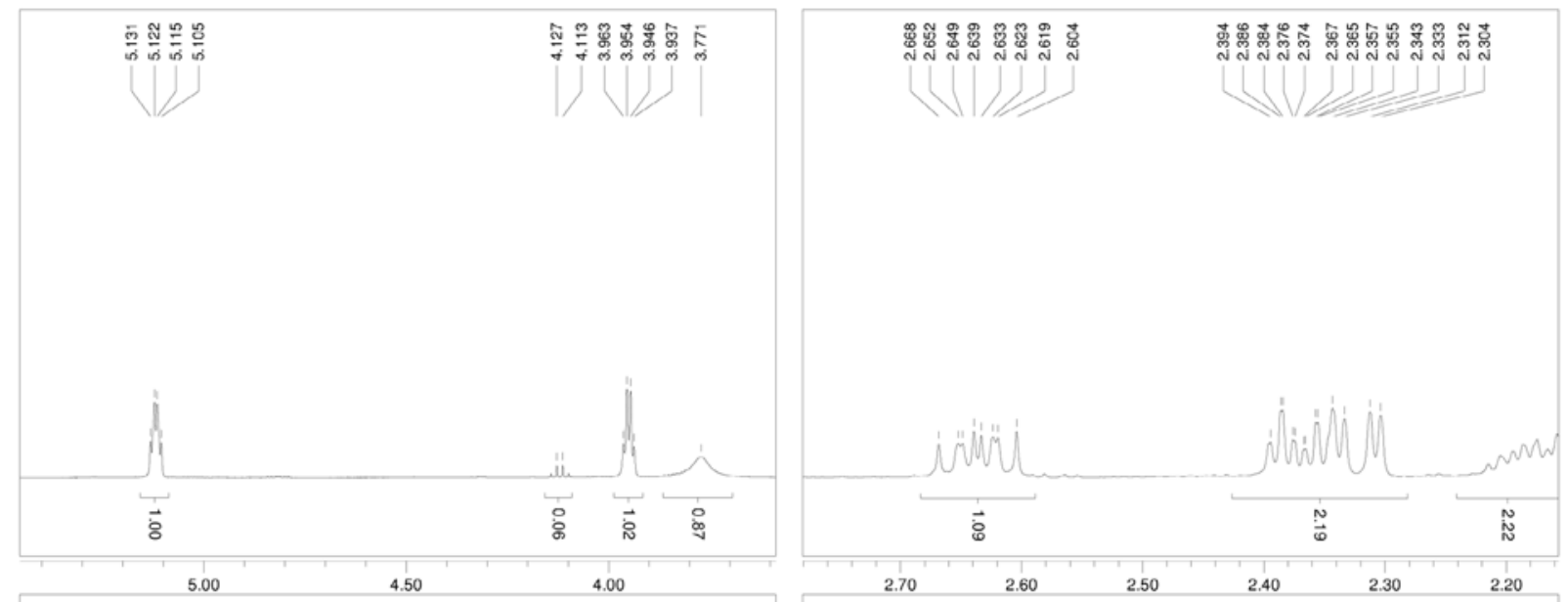

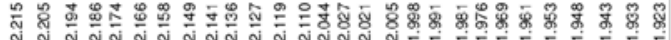

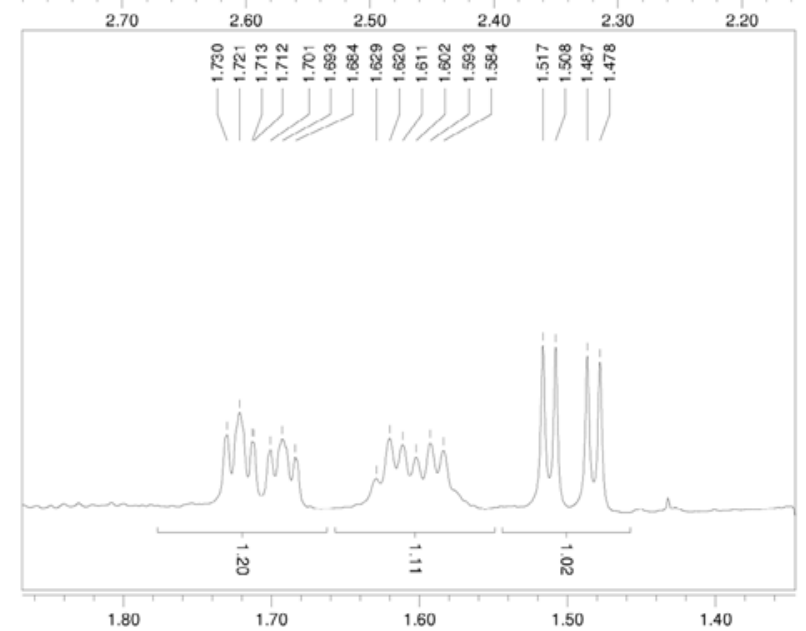

Figura 11S. Expansões do espectro de RMN de ${ }^{l} \mathrm{H}(500 \mathrm{MHz}), \mathrm{TMS}, \mathrm{CDCl}_{3}-$ Produto 23 


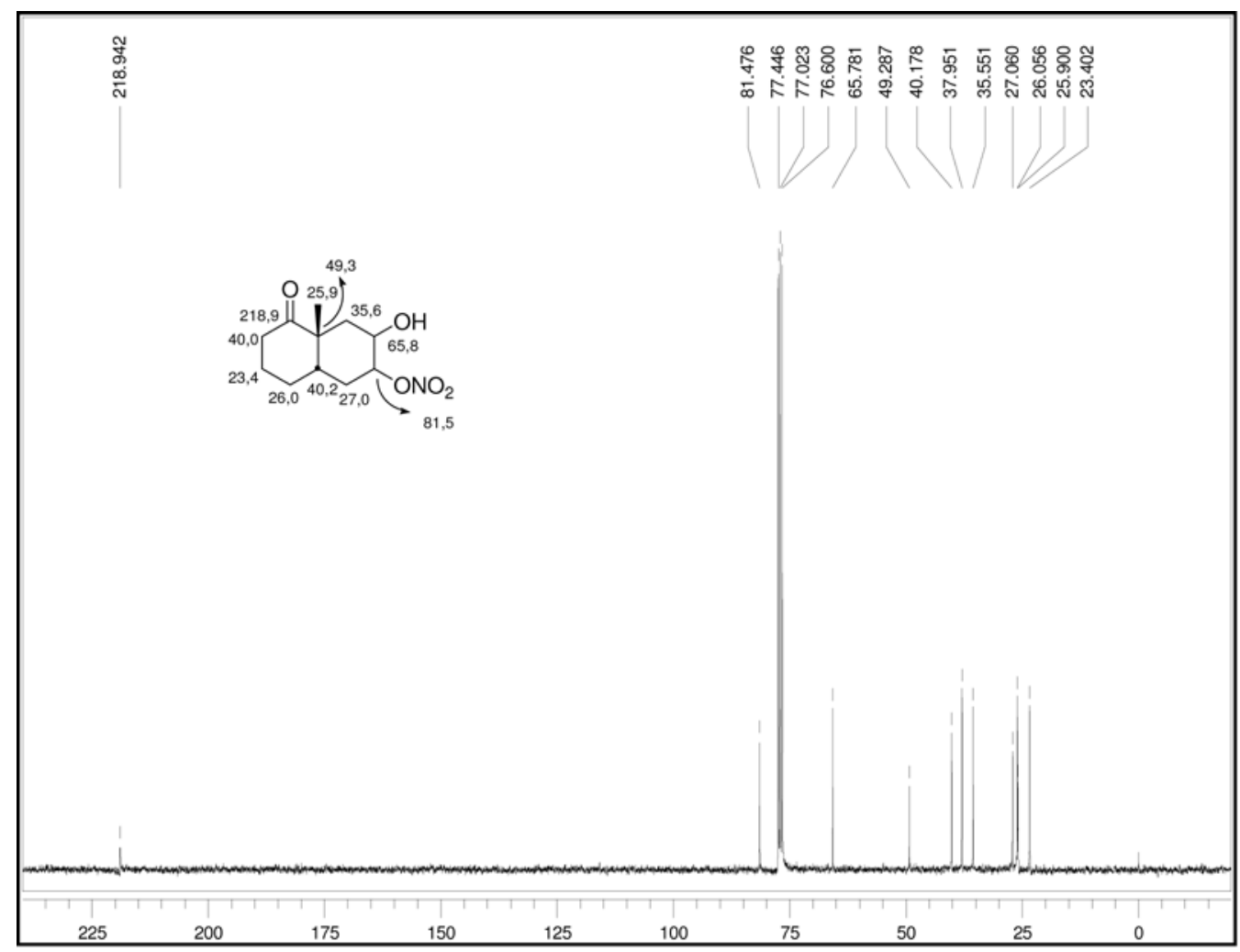

Figura 12S. Espectro de $\mathrm{RMN} \mathrm{de}{ }^{13} \mathrm{C}(75 \mathrm{MHz}), \mathrm{TMS}, \mathrm{CDCl}_{3}-$ Produto 23

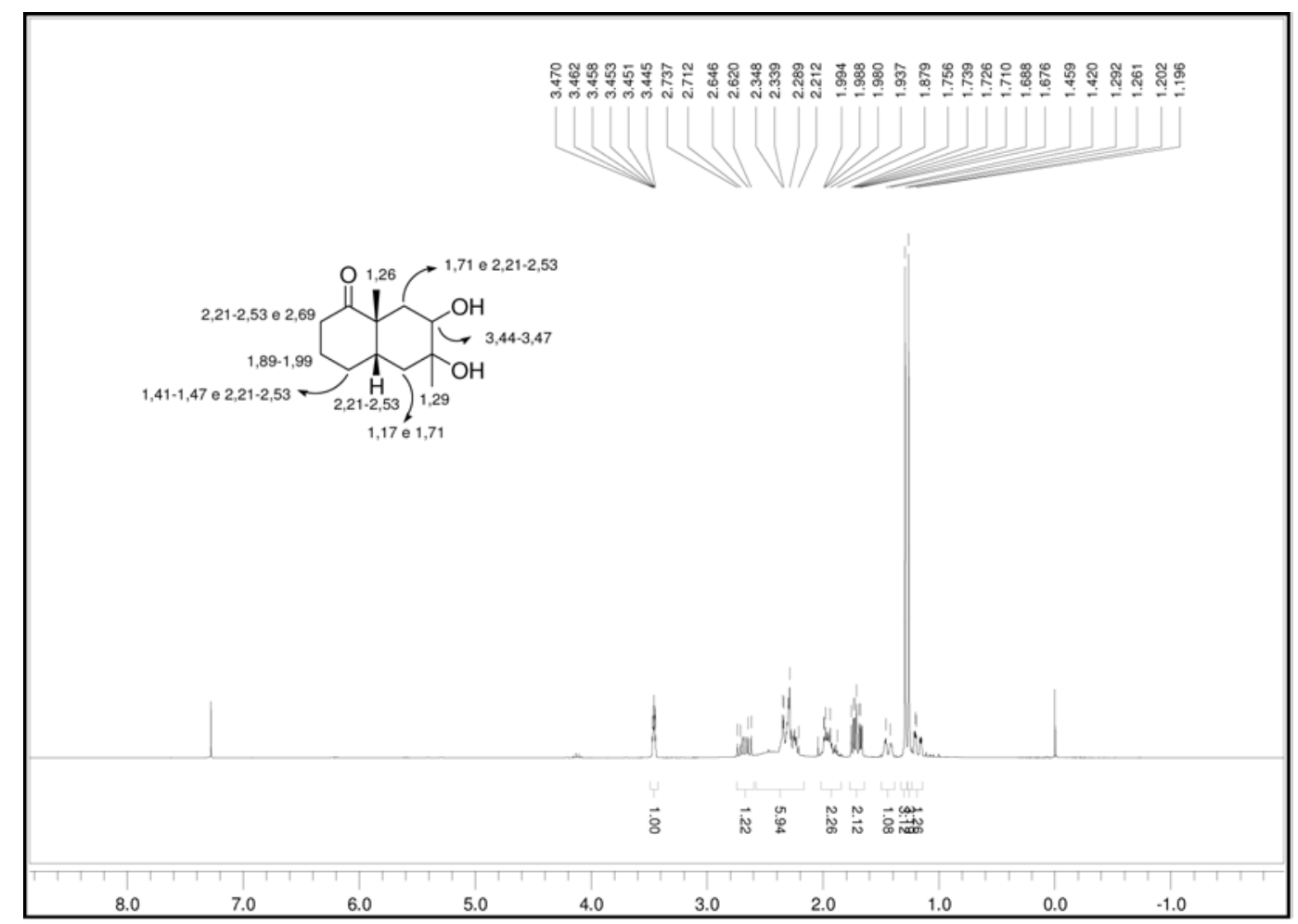

Figura 13S. Espectro de RMN de ${ }^{l} \mathrm{H}(300 \mathrm{MHz}), \mathrm{TMS}, \mathrm{CDCl}_{3}-$ Produto 24 


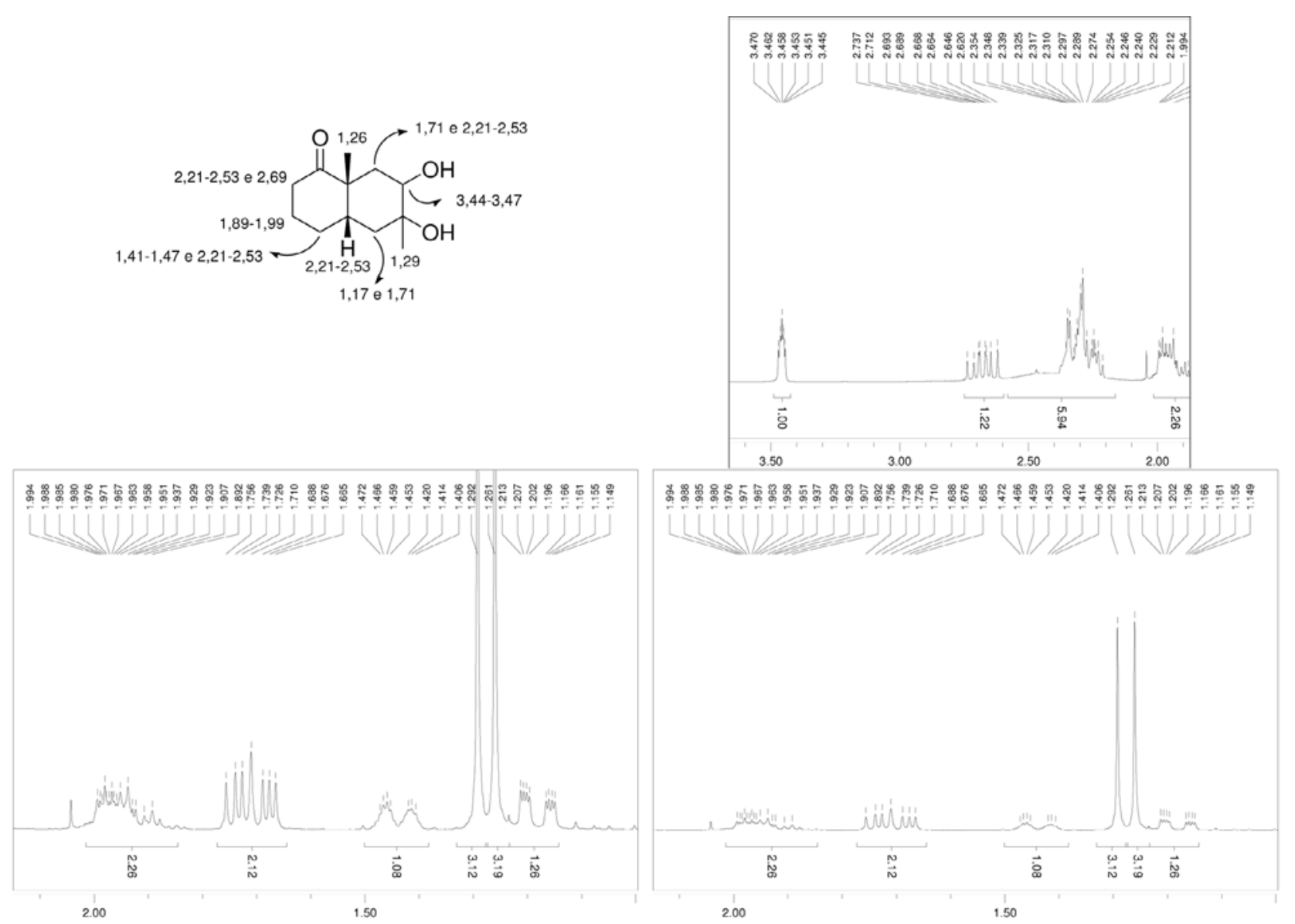

Figura 14S. Expansões do espectro de $\mathrm{RMN}$ de ${ }^{1} \mathrm{H}(300 \mathrm{MHz})$, TMS, $\mathrm{CDCl}_{3}$ - Produto 24

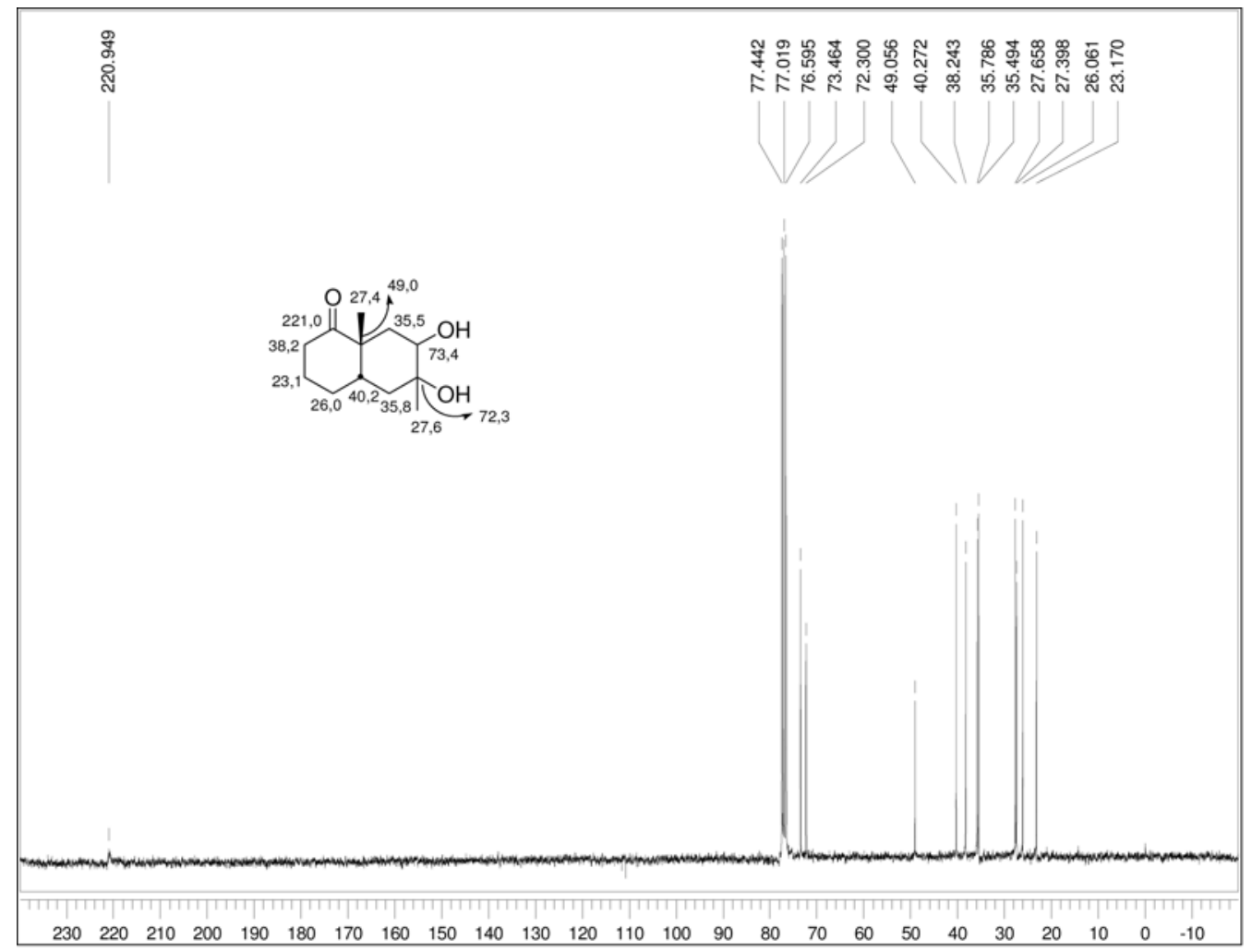

Figura 15S. Espectro de $\mathrm{RMN}$ de ${ }^{13} \mathrm{C}(75 \mathrm{MHz}), \mathrm{TMS}, \mathrm{CDCl}_{3}$ - Produto 24 